Cahiers
de la Recherche
surles Droits Fondamentaux

\section{Cahiers de la recherche sur les droits} fondamentaux

15 | 2017

Le corps humain saisi par le droit : entre liberté et propriété

\title{
Concept, genèse et évolution de l'amparo : le modèle espagnol
}

Concept, Genesis and Evolution of the Amparo: The Spanish Model

\section{Carlos Ruiz Miguel}

\section{CpenEdition}

\section{Journals}

Édition électronique

URL : https://journals.openedition.org/crdf/567

DOI : $10.4000 / \mathrm{crdf} .567$

ISSN : 2264-1246

Éditeur

Presses universitaires de Caen

Édition imprimée

Date de publication : 1 novembre 2017

Pagination : 133-152

ISBN : 978-2-84133-858-0

ISSN : 1634-8842

Référence électronique

Carlos Ruiz Miguel, « Concept, genèse et évolution de l'amparo : le modèle espagnol », Cahiers de la recherche sur les droits fondamentaux [En ligne], 15 | 2017, mis en ligne le 01 octobre 2019, consulté le 15 novembre 2022. URL : http://journals.openedition.org/crdf/567 ; DOI : https://doi.org/10.4000/crdf 567 


\title{
Concept, genèse et évolution de l'amparo: le modèle espagnol
}

\author{
Carlos RUIZ MIGUEL \\ Professeur de droit constitutionnel à l'université de Saint-Jacques de Compostelle (Espagne)
}

I. Préalable conceptuel: proposition d'une définition de l'amparo

II. Antécédents et développements en droit constitutionnel comparé et droit international
A. Antécédents
B. Consécration constitutionnelle de l'amparo
1. Mexique et Amérique
2. Espagne, Allemagne et reste de l'Europe
C. L'amparo en droit international des droits de l'homme

III. Le modèle espagnol: amparo ordinaire et amparo constitutionnel

A. L'amparo ordinaire

B. L'amparo constitutionnel: principes et champ de protection
1. Les principes de l'amparo constitutionnel
2. Le champ de protection de l'amparo constitutionnel

IV. Légitimation du recours d'amparo

V. L'objet de l'amparo

VI. La modification de la recevabilité du recours d'amparo et la mutation de sa nature

VII. La procédure et l'arrêt du recours d'amparo

VIII. Le recours d'amparo dans la politique constitutionnelle

L'amparo est l'institution constitutionnelle d'origine hispanique la plus répandue dans le monde, même si le monde germanique dispose d'une institution semblable. L'amparo a connu un vrai succès mais il faut distinguer cette figure d'autres institutions proches. Cette étude permettra d'examiner la notion (I), les origines et le développement du mécanisme (II), puis sa configuration en Espagne où l'on trouve un amparo pour les cours ordinaires et un autre pour la Cour constitutionnelle (III), dont les contours et problèmes (IV-VIII) seront ici examinés en prenant dûment en compte les données quantitatives liées à son application pratique.

\section{Préalable conceptuel: proposition d'une définition de l'amparo}

On peut définir l'amparo comme une « une procédure judiciaire particulière destinée à la protection des droits fondamentaux», composée stricto sensu de trois éléments. 
Primo, l'amparo protège les «droits fondamentaux». Par «droit fondamental», nous entendons un droit subjectif garanti dans la norme fondamentale ${ }^{1}$. Il n'y a pas d'amparo s'il n'y pas de protection d'un vrai droit subjectif et si ledit droit n'est pas établi dans la norme fondamentale. Secundo, ce droit fondamental doit bénéficier d'une «garantie judiciaire». Les droits fondamentaux jouissent de toute une série de garanties (réserve de loi, Ombudsman, tutelle judiciaire, etc.) mais ce n'est que lorsque le droit fondamental est protégé par une garantie judiciaire que l'on peut parler d'amparo. Une troisième condition est encore nécessaire pour définir l'amparo: cette garantie judiciaire doit être «spécifique». Aux États-Unis, il ne fait aucun doute que les droits fondamentaux sont protégés par les cours (judicial review). Mais la procédure judiciaire de cette protection est la même pour connaître de n'importe quelle autre affaire. Ce qui fait la singularité de l'amparo est que la protection des droits fondamentaux doit se faire au moyen d'une procédure spécifique, différente des autres procédures judiciaires existantes. Cette spécificité peut renvoyer à plusieurs situations:

- une garantie judiciaire spécifique pour certains droits fondamentaux, tout en excluant les autres droits fondamentaux;

- une garantie judiciaire spécifique déterminée par une spécialité procédurale. À cet égard, la spécialité peut consister en une exigence particulière pour la recevabilité de l'affaire (subsidiarité), un délai spécifique pour la résolution de l'affaire (procédure urgente), ou bien un jugement avec des effets spécifiques (effet limité aux particuliers parties dans l'affaire lorsque l'on établit l'annulation d'une norme générale).

\section{Antécédents et développements en droit constitutionnel comparé et droit international}

\section{A. Antécédents}

Il est possible de trouver quelques précédents à l'amparo, aussi bien dans la période pré-constitutionnelle qu'après l'ère du constitutionnalisme.
Plusieurs auteurs considèrent comme antérieure à l'amparo une institution dénommée Justicia Mayor ${ }^{2}$, qui existait au Moyen Âge espagnol dans le royaume d'Aragon. Elle visait à protéger les individus dans l'exercice de leurs libertés (qui à cette époque n'étaient pas encore des «droits fondamentaux»), tant auprès des autorités publiques (le roi, les nobles) que face à d'autres particuliers, et ceci que l'atteinte aux libertés soit actuelle ou future. Cette institution a cessé d'exister de facto en 1592 à la suite d'une crise politique majeure liée à la volonté du Justicia Mayor d'Aragon de protéger Antonio Pérez, le secrétaire d'État qui avait trahi le roi Philippe II. Sa fin de jure n'est cependant attestée qu'en $1707^{3}$.

D'autres auteurs évoquent un autre précédent de l'amparo dans l'Amérique espagnole. Après la découverte et la conquête du Nouveau Monde, on y a découvert des procédures déjà dénommées amparo. En Nouvelle Espagne (l'actuel Mexique), des affaires d'amparo étaient instruites, notamment sur des questions de propriété, par le vice-roi, soit directement soit indirectement en tant que président du tribunal suprême du territoire, la Real Audiencia de Mexico ${ }^{4}$. Le Pérou connaissait une autre procédure d'amparo « colonial $»^{5}$.

Certes, le constitutionnalisme américain est le premier à fournir une garantie judiciaire des droits constitutionnels. Le discours de James Madison en 1789, lors de sa proposition d'adoption de plusieurs amendements à la Constitution américaine de 1787 , est clair: pour lui, les tribunaux de justice indépendants seront les protecteurs des droits une fois ceux-ci incorporés à la Constitution. Mais cette judicial review ne s'insère pas dans une procédure spéciale différente de celle employée pour défendre quelqu'autre droit ou intérêt ${ }^{6}$.

On ne peut pas davantage trouver un précédent ${ }^{7}$ dans le projet de Sieyès de 1795 de «jurie constitutionnaire» dont l'une des fonctions était d'être un «tribunal des droits de l'homme ${ }^{8}$. Un examen attentif nous montre en effet qu'il n'existe pas d'amparo dans la proposition de Sieyès. Cette «jurie constitutionnaire» est un "supplément de juridiction naturelle aux vides de la juridiction positive» 9 , car il s'agit de «donner à un jugement d'équité naturelle la force obligatoire qu'ont les sentences ordinaires de nos

1. Voir O. Pfersmann, «Esquisse d'une théorie des droits fondamentaux en tant qu'objets juridiques", in Droit des libertés fondamentales, L. Favoreu et al. (dir.), 6 éd., Paris, Dalloz, 2012, p. 63 sq. (spéc. p. 75)

2. Voir R. Reyes, La defensa constitucional, Madrid, Espasa-Calpe, 1934, p. 25-30; V. Fairén Guillén, «La defensa del derecho de libertad personal en la historia y en la actualidad españolas», Revista de administración pública, n 69, 1972, p. 9 sq.; V. Fairén Guillén, «La defensa procesal de la libertad y dignidad personales en una futura Constitución española », Revista de administración pública, n 83, 1977, p. 9 sq.; G. Eto Cruz, «La herencia constitucional española en la formación del amparo en Latinoamérica » (texte consulté en ligne mais qui n’est désormais plus accessible).

3. Ordonnance royale (decreto) d'abolition des juridictions (fueros) d'Aragon et Valence, 29 juin 1707.

4. Voir A. Lira González, El amparo colonial y el juicio de amparo mexicano, Mexico, Fondo de cultura económica, 1972, p. 16 sq.

5. Voir G. Eto Cruz, Tratado del proceso constitucional de amparo, Lima, Gaceta Jurídica, 2013, t. I, p. 51-68.

6. "If they are incorporated into the constitution, independent tribunals of justice will consider themselves in a peculiar manner the guardians of those rights" ("Madison Speech Proposing the Bill of Rights June 8 1789», in The Debates and Proceedings in the Congress of the United States, J. Gales (éd.), Washington, Gales and Seaton, 1834, vol. I, p. 448-459, spéc. p. 457).

7. Lucien Jaume n'affirme pas cette thèse mais en laisse la porte ouverte. Voir L. Jaume, «Sieyès et le sens du jury constitutionnaire: une réinterprétation », Droits, vol. 36, n' 2, 2002, p. 115-134.

8. E. J. Sieyès, Opinion sur la jurie constitutionnaire, s.l.s.n., 1795, p. 29

9. Ibid., p. 5 
tribunaux ${ }^{10}$. Sieyès prétend ici trouver une substitution à l'ancien «droit de grâce ${ }^{11}$ considéré comme une prérogative royale.

Le précédent le plus important de l'amparo semble en réalité se trouver dans les origines du constitutionnalisme espagnol. L'institution du Justicia Mayor fut bel et bien évoquée aux Cortes qui avaient élaboré la Constitution de Cadix de 1812, même si une telle institution n'avait pas été reprise dans le texte. Mais les Cortes dans leur législation complémentaire à la Constitution avaient approuvé un bref mais extrêmement important décret, sept mois après avoir approuvé la Constitution le 19 mars 1812. Il s'agit d'un texte qui, au-delà de prévoir une garantie judiciaire pour la Constitution, établit que lesdites procédures doivent être examinées avec plus de célérité que les autres. Nous trouvons bien ici une singularité, la «préférence». Même si cette procédure judiciaire n'est pas uniquement établie pour connaître des affaires relevant des droits constitutionnels, il ne fait aucun doute que les affaires concernant ces droits relèvent de cette procédure ${ }^{12}$.

\section{B. Consécration constitutionnelle de l'amparo}

\section{Mexique et Amérique}

C'est au Mexique, quelques années après être devenu indépendant de l'Espagne, que l'on trouve la naissance d'une institution particulière de protection des droits constitutionnels appelée «amparo». Cette institution est le résultat de l'influence de trois facteurs: le droit colonial; les débats sur la Constitution espagnole de Cadix de 1812 (connue des députés mexicains qui sont intervenus dans son élaboration) et la législation des Cortes $^{13}$; et le droit nord-américain (la judicial review) ${ }^{14}$.

Le premier texte constitutionnel du monde mentionnant l'amparo est la Constitution de l'État fédéré du Yucatan de 1841, avec l'inspiration directe du juriste Manuel Crescencio Rejón, qui prévoit explicitement une procédure judiciaire spéciale pour la protection des droits constitutionnels (c'est-à-dire fondamentaux) ${ }^{15}$. Six ans plus tard, l'amparo s'étend à toute la Fédération mexicaine lorsqu'est approuvée la loi sur les réformes (de la Constitution fédérale de 1824) promulguée en $1847^{16}$. Cette loi fut rédigée par le juriste Mariano Otero (d'où l'appellation «formule Otero" pour cette construction de l'amparo). D'après la «formule Otero", l'obtention de l'amparo par le juge bénéficie seulement au plaignant et n'a pas de portée générale, même si l'on déclare que c'est la norme générale elle-même qui est inconstitutionnelle. La configuration mexicaine se retrouve dans la Constitution fédérale du 5 février $1857^{17}$. L'amparo est également reconnu dans la Constitution de 1917 (qui, techniquement, n'est qu'une révision de la Constitution de 1857) qui reprend le concept tel qu'il ressort de cette évolution. Si l'on veut faire une synthèse du contenu de l'institution de l'amparo en droit mexicain, il suffit d'indiquer qu'il permet à tout citoyen de saisir les tribunaux fédéraux de toute norme particulière (qu'elle émane de l'exécutif ou du judiciaire) ou générale directement applicable (qu'elle émane de l'exécutif ou du législatif) considérée comme préjudiciable à certains droits constitutionnels du plaignant. Notamment, pour la Constitution de 1917 d'après l'article 103.1:

Les tribunaux fédéraux seront chargés de résoudre toute controverse qui dérive [...] des lois ou d'actes émanant d'une autorité, tant que leur mise en place entraîne une transgression aux garanties individuelles [garanties reconnues dans les articles 1-29 de la Constitution] ${ }^{18}$.

L'octroi de l'amparo emporte inapplication de la norme (particulière ou générale) mais ses effets sont simplement inter partes. Les décisions d'octroi de l'amparo non seulement annulent (pour le requérant) l'acte ou la norme préjudiciable, mais encore elles ont force de condamnation. En effet, si l'atteinte a pour origine une action positive, l'amparo peut constituer une injonction

10. Ibid., p. 24

11. Ibid., p. 25

12. Décret CCX, 28 novembre 1812: «Que los tribunales prefieran á otros negocios los relativos á infracción de Constitución. / Las Córtes generales y extraordinarias, convencidas de la necesidad y utilidad de que los expedientes sobre infracción de la Constitución sean determinados con la mayor prontitud, decretan: Los Tribunales del Reino preferirán á todo otro asunto los relativos á infracción de la Constitución política de la Monarquía [...]».

13. Voir V. Fairén Guillén, «La defensa del derecho... », p. 44-45.

14. Voir H. Fix-Zamudio, Ensayos sobre el derecho de amparo, Mexico, UNAM, 1993, p. 23.

15. «Artículo 8. Los jueces de primera instancia ampararán en el goce de los derechos garantidos por el artículo anterior, a los que les pidan su protección contra cualesquier funcionarios que no correspondan al orden judicial, decidiendo brevemente y sumariamente las cuestiones que se susciten sobre los asuntos indicados. / Artículo 9. De los atentados cometidos por los jueces contra los citados derechos, conocerán sus respectivos superiores con la misma preferencia de que se ha hablado en el artículo precedente; remediando desde luego el mal que se les reclame, y enjuiciando inmediatamente al conculcador de las mencionadas garantías."

16. "Acta constitutiva y de reformas de 1847", 21 mai 1847: "Artículo 25. Los tribunales de la Federación ampararán a cualquiera habitante de la República, en el ejercicio y conservación de los derechos que le concedan esta Constitución y las leyes constitucionales, contra todo ataque de los poderes legislativo y ejecutivo, ya de la Federación, ya de los Estados; limitándose dichos tribunales a impartir su protección en el caso particular sobre que verse el proceso, sin hacer ninguna declaración general respecto de la ley o del acto que lo motivare».

17. «Artículo 101. Los tribunales de la federación resolverán toda controversia que se suscite: I. Por leyes ó actos de cualquier autoridad que violen las garantías individuales. II. Por leyes ó actos de la autoridad federal que vulneren ó restrinjan la soberanía de los Estados. III. Por leyes ó actos de las autoridades de éstos, que invadan la esfera de la autoridad federal. / Article 102. Todos los juicios de que habla el artículo anterior se seguirán, á petición de la parte agraviada, por medio de procedimientos y formas del orden jurídico, que determinará una ley. La sentencia será siempre tal, que solo se ocupe de individuos particulares, limitándose á protegerlos y ampararlos en el caso especial sobre que verse el proceso, sin hacer ninguna declaración general respecto de la ley ó acto que la motivare."

18. «Los tribunales de la Federación resolverán toda controversia que se sucite [...] por leyes o actos de la autoridad que viole las garantías individuales» (sauf mention contraire, toutes les traductions de l'espagnol sont de moi). 
à l'adresse de l'autorité défenderesse afin que les choses reviennent en leur état antérieur, et si elle a pour origine une omission, les autorités défenderesses doivent satisfaire aux dispositions législatives ou constitutionnelles ${ }^{19}$.

Le succès de l'amparo en Amérique est retentissant. Presque tous les pays ibero-américains ont consacré une procédure judiciaire spéciale pour la protection des droits fondamentaux. Tel est le cas de l'Argentine ${ }^{20}$, de la Bolivie ${ }^{21}$, du Brésil (mandado de segurança) ${ }^{22}$, de la Colombie (acción de tutela) ${ }^{23}$, du Costa Rica ${ }^{24}$, du Chili (recurso de protección $)^{25}$, de l'Équateur ${ }^{26}$, du Salvador ${ }^{27}$, du Guatemala ${ }^{28}$, du Honduras $^{29}$, du Nicaragua ${ }^{30}$, du Panama ${ }^{31}$, du Paraguay ${ }^{32}$, de l'Uruguay ${ }^{33}$ et du Venezuela ${ }^{34}$.

\section{Espagne, Allemagne et reste de l'Europe}

L'amparo fait sa première apparition en Europe dans la Constitution espagnole de 1931, mais on peut aussi faire référence à deux textes de l'espace germanique qui avaient balisé le terrain pour une future réception de l'amparo: la Constitution allemande de 1849 et la Constitution autrichienne de 1920 après sa réforme de 1929.

En 1849, deux ans après la consécration de l'amparo dans la Constitution fédérale mexicaine, la Constitution allemande de l'église Saint-Paul (28 mars 1849) va ouvrir la porte (porte que la réaction anti-révolutionnaire va tout de suite fermer) à une protection des droits constitutionnels qui pouvait aussi bien être façonnée sur le modèle des
États-Unis (judicial review) que sur celui du Mexique (amparo). Cette Constitution consacre la protection judiciaire des droits constitutionnels mais laisse à la législation la concrétion de la forme et du mode (Art und Weisen) dont on peut faire l'usage ${ }^{35}$.

Quelques décennies plus tard, en Autriche, la réforme du 7 décembre 1929 de la Constitution de 1920 introduisait un recours individuel auprès de la Cour constitutionnelle ${ }^{36}$. Cette réforme permet d'introduire un recours devant la Cour constitutionnelle contre les décisions des autorités dans la mesure où le plaignant prétend avoir été lésé par la violation d'un droit constitutionnel ou par l'application d'un règlement non conforme à la loi, d'une loi inconstitutionnelle ou d'un traité international non conforme au droit. Le recours ne peut être introduit qu'après épuisement des voies de recours ordinaires. Il s'agit d'une procédure très proche de l'amparo mais qui ne peut pas être qualifiée comme telle car elle n'est pas réservée à la protection des droits constitutionnels, étant donné que la même procédure peut être employée pour contester la légalité d'un règlement administratif.

La première réception de l'amparo en Europe se trouve en Espagne dans sa Constitution de 1931. Deux ans auparavant, en 1929, le gouvernement du dictateur Primo de Rivera avait rédigé un avant-projet de Constitution qui prévoyait un contrôle de la constitutionnalité des lois qui pourrait être déclenché par l'individu et dont les

19. Voir H. Fix-Zamudio, Ensayos sobre el derecho de amparo, p. 64.

20. Art. 43, al. 1 et 2 de la Constitution de 1853 (introduit dans la dernière réforme en 1994) et loi no 16986 , Ley de Acción de Amparo du 18 octobre 1966.

21. Art. 128 sq. de la Constitution de 2009 et loi $\mathrm{n}^{\circ} 027$ du 6 juillet 2010.

22. Art. 5, al. LXIX et LXX de la Constitution de 1988 et loi $\mathrm{n}^{\circ} 12.016$ du 7 août 2009.

23. Art. 86 de la Constitution de 1991 et décret nº 2591 du 9 novembre 1991.

24. Art. 48 de la Constitution de 1949 et loi $n^{\circ} 7135$ du 10 octobre 1989.

25. Art. 20 de la Constitution de 1980, développé par l'auto acordado 94-2015 du 27 septembre 2015 de la Cour constitutionnelle.

26. Art. 88 de la Constitution de 2008 et loi organique relative aux garanties judiciaires et au contrôle constitutionnel du 21 septembre 2009 .

27. Art. 247 de la Constitution de 1983 et décret no 2996 portant loi des procédures constitutionnelles du 14 janvier 1960 . L'amparo au Salvador est reconnu au niveau constitutionnel par l'article 37 de la Constitution du 13 août 1886. Voir M. Montecino Giralt, «El amparo en El Salvador: finalidad y derechos protegibles", Revista IUS, vol. 5, $\mathrm{n}^{\circ} 27,2011, \mathrm{p} .126-144$.

28. Art. 265 de la Constitution de 1993 et décret no $1-86$ du 8 janvier 1986 portant loi d'amparo.

29. Art. 183 de la Constitution de 1982 et loi sur la justice constitutionnelle du 20 janvier 2004. L'article 29 de la Constitution de 1894 prévoyait déjà l'amparo.

30. Art. 45 et 188 de la Constitution de 1986 et loi no 49 du 16 novembre 1988, loi d'amparo.

31. Art. 54 de la Constitution de 1972: l'amparo est développé dans le titre III du quatrième livre (art. 2615-2632) du Code judiciaire, approuvé par la résolution $\mathrm{n}^{\circ} 1$ du 30 août 2001. L'amparo est reconnu au Panama depuis la Constitution de 1941 (art. 189).

32. Art. 134 de la Constitution de 1992. La réglementation de l'amparo est développée dans le titre II du cinquième livre (art. 565-588) du Code de procédure civile, loi n ${ }^{\circ} 1.337$ du 4 novembre 1988. C'est dans la Constitution de 1967 que l'on constitutionnalise pour la première fois l'amparo.

33. La Constitution de l'Uruguay (de 1967, modifiée en 2004) ne prévoit pas l'institution de l'amparo, mais elle est instaurée par la loi nº 16.011 (Ley de acción de amparo) du 7 décembre 1988.

34. Art. 27 de la Constitution de 1999, loi organique d'amparo sur les droits et les garanties constitutionnelles du 27 septembre 1988 , amendée le 22 juillet 2014.

35. «\$ 126. Zur Zuständigkeit des Reichsgerichts gehören: $[\ldots] \mathrm{g}$ ) Klagen deutscher Staatsbürger wegen Verletzung der durch die Reichsverfassung ihnen gewährten Rechte. Die näheren Bestimmungen über den Umfang dieses Klagerechts und die Art und Weise dasselbe geltend zu machen, bleiben der Reichsgesetzgebung vorbehalten.»

36. "Artikel 144. (1) Der Verfassungsgerichtshof erkennt über Beschwerden gegen Bescheide (Entscheidungen und Verfügungen) der Verwaltungsbehörden, soweit der Beschwerdeführer durch den Bescheid in einem verfassungsgesetzlich gewährleisteten Recht verletzt zu sein behauptet. Die Beschwerde kann, sofern bundesgesetzlich nicht anderes bestimmt, erst nach Erschöpfung des Instanzenzuges erhoben werden. / (2) Die Bestimmungen des Artikels 133, Absätze 1 und 3, gelten in diesen Fällen sinngemäß für das Erkenntnis des Verfassungsgerichtshofes. / (3) Findet der Verfassungsgerichtshof, daß durch den angefochtenen Bescheid der Verwaltungsbehörde ein verfassungsgesetzlich gewährleistetes Recht nicht verletzt wurde, und handelt es sich nicht um einen Fall, der nach Art. 129, Absatz 5, von der Zuständigkeit des Verwaltungsgerichtshofes ausgeschlossen ist, so hat der Verfassungsgerichtshof zugleich mit dem abweisenden Erkenntnis auf Antrag des Beschwerdeführers die Beschwerde zur Entscheidung darüber, ob der Beschwerdeführer durch den Bescheid in einem sonstigen Recht verletzt wurde, dem Verwaltungsgerichtshof abzutreten.» 
effets d'un éventuel arrêt d'inconstitutionnalité seraient immédiatement exécutifs pour le plaignant ${ }^{37}$.

C'est dans ce contexte que l'amparo est introduit en 1931 en Espagne. Mais l'élément décisif pour l'inclusion de l'amparo dans la Constitution vient de la présence en Espagne d'un intellectuel mexicain exilé à Madrid et bien placé parmi les élites politiques et intellectuelles: Rodolfo Reyes. C'est lui qui avait diffusé l'institution de l'amparo même avant l'élection de l'Assemblée constituante de $1931^{38}$. Cependant, l'influence mexicaine passera par le filtre d'une autre source, non moins importante, qui lui donnera sa configuration définitive, à savoir l'apport kelsénien préconisant l'instauration d'un organe, séparé du pouvoir judiciaire, qui assume le monopole du contrôle de constitutionnalité. Le mélange des apports mexicain et autrichien prend corps dans une configuration de l'amparo qui sera différente du modèle mexicain. Ces deux éléments figurent dans l'article 121.b de la Constitution de 1931 qui institue l'amparo comme l'une des compétences du Tribunal des garanties constitutionnelles, créé pour la première fois en Espagne sur le modèle autrichien ${ }^{39}$.

Après la Seconde Guerre mondiale, c'est d'abord en Allemagne (1949) que l'on consacre une procédure inspirée de l'amparo, puis en Espagne (1978), et ce même si la doctrine allemande ne reconnaît pas d'influences hispaniques ${ }^{40}$. La Loi fondamentale de Bonn, dans sa première version du 23 mai 1949, n'avait pas prévu explicitement que la Cour constitutionnelle puisse statuer sur des amparo. Mais elle permettait cependant à la loi, dans son article 93.2, d'élargir les compétences de la Cour. C'est ainsi que l'amparo (Verfassungsbeschwerde ou plainte constitutionnelle) fut introduit dans la loi sur la Cour constitutionnelle de 1951. Quelques années plus tard, le 29 janvier 1969, la Loi fondamentale de Bonn fut amendée pour inclure la Verfassungsbeschwerde parmi les compétences de la Cour. La Constitution allemande reconnaît alors pour la première fois explicitement compétence à la Cour constitutionnelle pour être saisie des recours constitutionnels qui peuvent être formés par quiconque estime avoir été lésé par la puissance publique dans l'un de ses droits fondamentaux, ou dans l'un de ses droits garantis par les articles 20, alinéas 4, 33, 38,101, 103 et 104 de ladite Loi fondamentale ${ }^{41}$.

En 1978, la Constitution espagnole a décidé d'inclure l'amparo comme protection spécifique des droits fondamentaux. L'amparo mexicain, la Constitution précédente de 1931 et le recours constitutionnel allemand (Verfassungsbeschwerde) étaient alors très présents dans les esprits. L'article 53.2 de la Constitution espagnole instaure deux mécanismes de protection des droits fondamentaux: d'une part, un recours d'amparo «ordinaire» ou "judiciaire", à la charge des tribunaux ordinaires (proche de l'amparo mexicain) et, d'autre part, un recours d'amparo «constitutionnel», confié au Tribunal constitutionnel (art. 161.b de la Constitution espagnole) dans la droite ligne de l'amparo prévu dans la Constitution de 1931 et de la Verfassungsbeschwerde allemande. La réglementation détaillée du recours d'amparo constitutionnel se trouve aux articles 41 et suivants de la loi organique du Tribunal constitutionnel.

Si jusqu'en 1990 l'Allemagne et l'Espagne étaient les seuls pays d'Europe à disposer d'une procédure d'amparo (en dehors du cas spécial de l'Autriche), la majorité des nouvelles démocraties de l'Europe de l'Est ont introduit l'amparo dans leur système constitutionnel après la chute du mur de Berlin. C'est le cas de la Croatie ${ }^{42}$, de la Macédoine ${ }^{43}$, de la Slovénie ${ }^{44}$, de la République tchèque ${ }^{45}$, de la Slovaquie ${ }^{46}$, de la Russie ${ }^{47}$, de la Pologne ${ }^{48}$. Les Constitutions de la Bosnie-Herzégovine ${ }^{49}$ et de la Hongrie $^{50}$ ouvrent la porte de la Cour constitutionnelle à l'individu, mais elles n'établissent pas une procédure spécifique pour la protection des droits fondamentaux. De même, on ne peut pas qualifier d'amparo la question prioritaire de constitutionnalité prévue à l'article 61-1

37. Avant-projet constitutionnel de 1929: «Art. 103. Toda infracción constitucional realizada individualmente por Ministros, autoridades, representantes o funcionarios de cualquiera especie, o colectivamente por los órganos o asambleas en que radiquen los diferentes Poderes, dará lugar a recursos judiciales. Estos recursos serán: [...] $/ 4^{\circ}$ El recurso por inconstitucionalidad de las leyes, que podrá interponerse en casos individuales y concretos de infracción constitucional. [...] / El fallo sobre inconstitucionalidad de una ley, será también inmediatamente ejecutivo en el caso particular para el que a instancia de parte legítima se hubiere dictado».

38. Ainsi en témoigne la lettre-prologue d'Angel Ossorio y Gallardo au livre de Rodolfo Reyes, La defensa constitucional, p. 10 (Ossorio y Gallardo fut ministre en 1920, député de la Constituante de 1931 et doyen du barreau de Madrid entre 1930 et 1933).

39. "Artículo 121. Se establece, con jurisdicción en todo el territorio de la República, un Tribunal de Garantías Constitucionales, que tendrá competencia para conocer de: [...] b) El recurso de amparo de garantías individuales, cuando hubiere sido ineficaz la reclamación ante otras autoridades.»

40. Voir P. Häberle, «Die Verfassunsbeschwerde im System der bundesdeutschen Verfassungsgerichtsbarkeit», Jahrbuch des öffentlichen Rechts, $\mathrm{n}^{\circ} 45,1997$, p. $89-136$.

41. "Art. 93. (1) Das Bundesverfassungsgericht entscheidet: [...]/4a. über Verfassungsbeschwerden, die von jedermann mit der Behauptung erhoben werden können, durch die öffentliche Gewalt in einem seiner Grundrechte oder in einem seiner in Artikel 20 Abs. 4, 33, 38, 101, 103 und 104 enthaltenen Rechte verletzt zu sein.»

42. Art. 129 de la Constitution de 1990.

43. Art. 50 de la Constitution de 1991.

44. Art. 160 de la Constitution de 1991.

45. Art. 87, al. 1.d de la Constitution de 1992.

46. Art. 127, al. 1 de la Constitution de 1992.

47. Art. 125, al. 4 de la Constitution de 1993.

48. Art. 79 de la Constitution de 1997.

49. Art. VI.3.b de la Constitution de 1995

50. Art. 24.2 de la Constitution de 2011. 
de la Constitution française de 1958, telle qu'elle a été introduite en 2008 , parce que la question peut seulement être posée par le Conseil d'État ou la Cour de cassation mais non par l'individu.

\section{L'amparo en droit international des droits de l'homme}

Au vu de ce qui a été déjà exposé, il n'est pas étonnant de voir que c'est en Amérique que l'on trouve la première consécration internationale de l'amparo. La Déclaration américaine des droits et devoirs de l'homme du 2 mai 1948, dans son article 18 (droit à la justice) est le premier document qui proclame un "droit» à l'amparo même si elle ne fait pas usage de cette dénomination ${ }^{51}$. Cette présentation contestable de l'amparo comme un «droit» et non comme une "garantie» des droits se trouve consacrée dans l'article 25 de la Convention américaine des droits de l'homme du 22 novembre $1969^{52}$. Cette configuration permet d'expliquer pourquoi l'amparo connaît une reconnaissance si étendue en Amérique. En effet, les États parties à la Convention de 1969 se sont engagés à mettre en œuvre dans leurs systèmes juridiques un «amparo» qui, de plus, est considéré comme un "droit de l'homme».

La Déclaration américaine de 1948 est la première proclamation internationale de l'histoire d'un catalogue des droits de l'homme. Il est tout à fait logique que ce texte ait eu une puissante influence sur les Nations unies au moment où celles-ci s'apprêtaient à proclamer un catalogue universel des droits de l'homme. En effet, l'influence de l'article 18 de la Déclaration américaine de 1948 est parfaitement visible dans l'article 8 de la Déclaration universelle des droits de l'homme ${ }^{53}$, même si la Déclara- tion universelle n'intègre pas l'exigence d'une procédure "simple et rapide», ce qui fait que ce «droit à un recours effectif» ne peut pas être qualifié d' "amparo" ${ }^{54}$.

Il existe une différence substantielle entre le système américain et le système des Nations unies. Si le premier maintient le contenu et améliore le statut juridique international de l'amparo, le deuxième va restreindre sa proclamation du "droit à un recours effectif». En effet, si la Déclaration universelle de 1948 parle d'un recours effectif «devant les juridictions nationales », l'article 2.3 $\mathrm{du}$ Pacte international des droits civils et politiques du 19 décembre 1966 parle d'un « recours utile» devant une autorité «judiciaire, administrative ou législative» ${ }^{55}$.

Le droit européen n'a pas reconnu d'obligation internationale d'établir une procédure d'amparo pour la protection des droits de l'homme, ni dans le cadre du Conseil de l'Europe ni dans celui de l'Union européenne (UE).

Il faut souligner que, malgré la proximité dans le temps de la Convention européenne des droits de l'homme (Convention EDH, 1950) et des Déclarations américaine et universelle des droits de l'homme (1948), l'instrument européen ne contient aucune référence au «droit» à l'amparo ou à une protection judiciaire des droits de l'homme comme cela avait été reconnu dans la Déclaration américaine (art. 18) ou universelle (art. 8). L'article 6.1 de la Convention EDH garantit le droit à ce que toute "cause " ayant un caractère civil ou pénal soit entendue par un tribunal ${ }^{56}$. En outre, la Cour a rappelé que «l'article 6 de la Convention n'astreint pas les États contractants à créer des juridictions d'appel ou de cassation et, encore moins, des juridictions compétentes en matière d'amparo ${ }^{57}$. Qui est plus, la Convention EDH ajoute dans son article 13 un "droit à un recours effectif» pour protéger les droits reconnus dans la Convention,

51. «Every person may resort to the courts to ensure respect for his legal rights. There should likewise be available to him a simple, brief procedure whereby the courts will protect him from acts of authority that, to his prejudice, violate any fundamental constitutional rights" [ "Toute personne peut recourir aux tribunaux pour faire valoir ses droits. De même, il doit exister une procédure simple et rapide qui permette à la justice de la protéger contre les actes de l'autorité violant, à son préjudice, certains droits fondamentaux reconnus par la Constitution»].

52. "Right to Judicial Protection: Everyone has the right to simple and prompt recourse, or any other effective recourse, to a competent court or tribunal for protection against acts that violate his fundamental rights recognized by the constitution or laws of the state concerned or by this Convention, even though such violation may have been committed by persons acting in the course of their official duties. / 2. The States Parties undertake: a. to ensure that any person claiming such remedy shall have his rights determined by the competent authority provided for by the legal system of the state; / $b$. to develop the possibilities of judicial remedy; and / c. to ensure that the competent authorities shall enforce such remedies when granted" [«Protection judiciaire: 1 . Toute personne a droit à un recours simple et rapide, ou à tout autre recours effectif devant les juges et tribunaux compétents, destiné à la protéger contre tous actes violant ses droits fondamentaux reconnus par la Constitution, par la loi ou par la présente Convention, lors même que ces violations auraient été commises par des personnes agissant dans l'exercice de fonctions officielles. / 2. Les États parties s'engagent: a. à garantir que l'autorité compétente prévue par le système juridique de l'État statuera sur les droits de toute personne qui introduit un tel recours;/b. à accroître les possibilités de recours judiciaire;/c. à garantir que les autorités compétentes exécuteront toute décision prononcée sur le recours»].

53. Déclaration universelle des droits de l'homme, A/RES/277 (III), adoptée à Paris le 10 décembre 1948

54. «Toute personne a droit à un recours effectif devant les juridictions nationales compétentes contre les actes violant les droits fondamentaux qui lui sont reconnus par la constitution ou par la loi.»

55. «Les États parties au présent Pacte s'engagent à: a) Garantir que toute personne dont les droits et libertés reconnus dans le présent Pacte auront été violés disposera d'un recours utile, alors même que la violation aurait été commise par des personnes agissant dans l'exercice de leurs fonctions officielles; / b) Garantir que l'autorité compétente, judiciaire, administrative ou législative, ou toute autre autorité compétente selon la législation de l'État, statuera sur les droits de la personne qui forme le recours et développer les possibilités de recours juridictionnel;/c) Garantir la bonne suite donnée par les autorités compétentes à tout recours qui aura été reconnu justifié.»

56. «Toute personne a droit à ce que sa cause soit entendue équitablement [...] par un tribunal [...] qui décidera, soit des contestations sur ses droits et obligations de caractère civil, soit du bien-fondé de toute accusation en matière pénale dirigée contre elle. [...]»

57. Cour EDH, 20 janvier 2015, Arribas Antón c. Espagne, n $16563 / 11, \$ 42$. 
mais ne consacre pas le caractère judiciaire dudit recours effectif ${ }^{58}$. En fait, dans sa première version de 1950, les individus n'avaient pas la capacité de porter une affaire devant la Cour européenne des droits de l'homme ${ }^{59}$. Ce n'est que depuis l'entrée en vigueur en 1998 du Protocole $n^{\circ} 11$ (signé en 1994) que la Cour peut être saisie par un particulier $^{60}$.

Dans le cadre de l'UE, un «amparo communautaire» a été proposé à l'article 38 du projet de Constitution européenne rédigé en 1994 par le Parlement européen ${ }^{61}$. Néanmoins, de lege lata, il n'existe pas d'amparo pour les droits fondamentaux en droit communautaire. D'après l'article 263.4 du Traité sur le fonctionnement de l'Union européenne, «[t]oute personne physique ou morale» peut saisir la Cour de justice de l'Union européenne pour contester n'importe quel acte ou norme communautaire, à condition que celle-ci le vise directement et individuellement ${ }^{62}$. Le Tribunal général de l'UE a interprété avec souplesse le concept de "personne morale», en considérant qu'une entité qui ne dispose pas de la personnalité juridique selon le droit d'un État membre ou d'un État tiers peut néanmoins être considérée comme une «personne morale», au sens de l'article 263.4, lorsque, dans leurs actes ou agissements, l'Union et ses institutions traitent l'entité en question comme étant un sujet distinct, qui peut posséder des droits qui lui sont propres ou être soumis à des obligations ou à des restrictions. Cela présuppose toutefois que l'entité en question dispose de statuts et d'une structure interne lui assurant l'autonomie nécessaire pour agir comme entité responsable dans les rapports juridiques ${ }^{63}$.

En Afrique, la déclaration du continent relative aux droits de l'homme ne consacre pas l'obligation d'introduire une procédure judiciaire spécifique pour la protection des droits de l'homme. Ainsi, l'article 26 de la Charte africaine des droits de l'homme et des peuples de 1981 exige seulement «l'établissement et le perfectionnement d'institutions nationales appropriées » à cet égard ${ }^{64}$. Néanmoins, le Protocole portant statut de la Cour africaine de justice et des droits de l'homme (2008) prévoit la possibilité pour toute personne relevant de la compétence d'un État partie de saisir la Cour africaine (Section des droits de l'homme) des affaires relatives à une violation alléguée d'un droit de l'homme ou des peuples ${ }^{65}$.

\section{Le modèle espagnol: amparo ordinaire et amparo constitutionnel}

Le modèle espagnol est caractérisé par l'existence de deux procédures d'amparo qui se trouvent connectées: l'amparo devant les cours ordinaires (amparo ordinaire) et l'amparo devant le Tribunal constitutionnel (amparo constitutionnel). Ce modèle est influencé par l'introduction du modèle d'amparo mexicain (dont le système judiciaire est inspiré des États-Unis, caractérisé par un contrôle diffus de la constitutionnalité) dans un système de contrôle de la constitutionnalité concentré inspiré du modèle autrichien.

\section{A. L'amparo ordinaire}

L'amparo ordinaire est prévu par l'article 53.2 de la Constitution espagnole de 1978 qui établit les trois principes qui lui confèrent sa spécificité: priorité, procédure sommaire et élargissement de l'intérêt à agir. La procédure sommaire vise à empêcher un abus de l'amparo puisqu'avec cette procédure il est uniquement possible de connaître des violations des droits fondamentaux, à l'exception de tout autre sujet. L'article 53.2 de la Constitution prévoit une autre spécificité pour cette procédure, son champ de protection, seuls les droits compris entre les articles 14

58. «Toute personne dont les droits et libertés reconnus dans la présente Convention ont été violés, a droit à l'octroi d'un recours effectif devan une instance nationale, alors même que la violation aurait été commise par des personnes agissant dans l'exercice de leurs fonctions officielles.»

59. Art. 44 de la première version de la Convention EDH: «Seules les Hautes parties contractantes et la Commission ont qualité pour se présenter devant la Cour».

60. Art. 34 de la Convention EDH (amendé par le Protocole $n^{\circ} 11$ ): «La Cour peut être saisie d'une requête par toute personne physique, toute organisation non gouvernementale ou tout groupe de particuliers qui se prétend victime d'une violation par l'une des Hautes Parties contractantes des droits reconnus dans la Convention ou ses protocoles. Les Hautes Parties contractantes s'engagent à n'entraver par aucune mesure l'exercice efficace de ce droit

61. Projet de Constitution européenne, 1994, Journal officiel des Communautés européennes, $\mathrm{n}^{\circ} \mathrm{C}$ 61, 28 février 1994: «Article 38 : Violation des droits de l'homme: La Cour de justice est compétente pour se prononcer sur tout recours introduit par un particulier visant à établir la violation par l'Union d'un droit de l'homme garanti par la Constitution. / Une loi constitutionnelle détermine les conditions d'introduction de ce recours et les sanctions que la Cour de justice peut décider».

62. Art. 263.4 du Traité sur le fonctionnement de l’Union européenne (ex-art. 230 du Traité instituant la Communauté européenne): «Toute personne physique ou morale peut former, dans les conditions prévues aux premier et deuxième alinéas, un recours contre les actes dont elle es le destinataire ou qui la concernent directement et individuellement, ainsi que contre les actes réglementaires qui la concernent directement et qui ne comportent pas de mesures d'exécution".

63. Arrêt du Tribunal (huitième chambre), 10 décembre 2015 dans l'affaire Front Polisario c. Conseil, T-512/12, $\$ 52$ et 53 .

64. «Les États parties à la présente Charte ont le devoir de garantir l'indépendance des Tribunaux et de permettre l'établissement et le perfectionnement d'institutions nationales appropriées chargées de la promotion et de la protection des droits et libertés garantis par la présente Charte.»

65. «Article 34: Introduction d'une instance devant la Section des droits de l'homme: 1. Les affaires portées devant la Cour relatives à une violation alléguée d'un droit de l'homme ou des peuples sont introduites par requête écrite adressée au Greffier. La requête doit indiquer le(s) droit(s) prétendument violé(s) ainsi que, dans la mesure du possible, la ou les dispositions de la Charte africaine des droits de l'homme et des peuples, de la Charte des droits et du bien-être de l'enfant, le Protocole à la Charte africaine des droits de l'homme et des peuples relatif aux droits des femmes en Afrique ou de tout autre instrument juridique relatif aux droits de l'homme, ratifié par l'État partie concerné, disposition(s) sur laquelle ou lesquelles il se fonde.» 
et 29 de la Constitution (mais tous ces droits) bénéficiant de la protection de l'action d'amparo devant les cours ordinaires.

La législation a prévu une autre spécificité de l'amparo ordinaire relative à l'intérêt à agir pour introduire une requête d'amparo. Le titulaire du droit fondamental lésé peut bien sûr ester en justice, mais le Procureur est partie dans toutes les procédures d'amparo ordinaire ${ }^{66}$. Il est ainsi toujours partie quand un amparo a été introduit par la partie lésée, mais lui aussi peut introduire une requête d'amparo en défense des droits fondamentaux ${ }^{67}$.

La responsabilité de protéger les droits fondamentaux appartient en premier lieu aux cours ordinaires. Cela explique l'existence d'une procédure d'amparo dans tous les ordres juridictionnels ordinaires (avec la seule exception de l'ordre pénal) : en matière civile ${ }^{68}$, en matière de contentieux administratif ${ }^{69}$, de contentieux électoral ${ }^{70}$, social $^{71}$ et militaire ${ }^{72}$. Pour les affaires de nature pénale, une seule procédure spécifique existe pour la protection d'un droit fondamental - le droit à la liberté personnelle - : l'habeas corpus ${ }^{73}$. On peut se demander pourquoi il n'existe pas un amparo en matière pénale alors que sont instaurés des amparo dans tous les autres ordres juridictionnels. L'explication suivante peut être avancée: lors de l'élaboration de la Constitution espagnole de 1978, un compromis avait été trouvé. Le droit de propriété (comme le voulait la droite) serait un droit constitutionnel, mais pas un droit à protection maximale (afin de satisfaire la gauche). Étant donné qu'un nombre substantiel d'affaires pénales affecte le droit de propriété (et donc l'économie), l'attribution d'un caractère prioritaire aux affaires concernant les droits fondamentaux aurait généré un retard substantiel pour les affaires concernant le droit de propriété et aurait entraîné des conséquences négatives pour la marche de l'économie. Refuser de donner un caractère prioritaire à de telles affaires aurait donc visé à éviter de tels préjudices.

\section{B. L'amparo constitutionnel: principes et champ de protection}

\section{Les principes de l'amparo constitutionnel}

Les principes fondant le recours d'amparo constitutionnel (RAC) sont au nombre de deux: la subsidiarité (avec l'exception logique de l'amparo contre acta interna corporis des Parlements) et son caractère réparateur (et non préventif). Ces deux principes ont été l'objet de quelques discussions doctrinales.

Le principe de subsidiarité est en cohérence avec l'existence d'un amparo ordinaire et avec l'attribution à la juridiction ordinaire de la compétence primaire de protection des droits fondamentaux. Mais si cette protection n'aboutit pas, on ouvre la porte de l'amparo constitutionnel. L'exception, tout à fait explicite, est celle de l'amparo contre des acta interna corporis des Parlements (national et régionaux) parce que ces actes ne peuvent être contestés devant aucune juridiction ordinaire. Le problème s'est posé autour de l'exigence de l'épuisement de toutes les voies de recours judiciaires ordinaires avant de saisir le Tribunal constitutionnel, conformément à une interprétation littérale de l'article 44.1.a de la loi organique du Tribunal constitutionnel (LOTC). Face à cette thèse s'est développée une autre interprétation fondée sur une interprétation systématique des articles 41.3, 49.1 et 54 de la LOTC. En vertu de cette lecture, il est nécessaire de prendre en compte les circonstances propres à chaque espèce en instance. La subsidiarité n'est alors pas un dogme sacro-saint, ni une règle à appliquer sans exceptions. Ainsi, face à une éventuelle violation "grave» des droits fondamentaux dont la préservation n'aurait pas été obtenue par la voie judiciaire, le RAC devrait être admis, sans attendre l'épuisement de toutes les voies de recours auprès des juges et des tribunaux. Il faudrait, dans ce cas, faire une distinction entre deux catégories de recours vues sous l'angle de leur éventuelle extemporanéité:

- ceux qui, sans avoir fait l'objet d'un jugement de révision par la voie judiciaire, sont introduits per saltum au Tribunal constitutionnel (et qui devraient être rejetés car prématurés);

- ceux qui visent des actes violant des droits fondamentaux de nature procédurale non susceptibles d'être réparés par le jugement définitif et qui ont mis fin à un incident ou à une demande incidente dans le cadre d'un procès principal, c'est-à-dire ceux au cours desquels les juges et les tribunaux n'ont pas saisi l'opportunité de préserver et de rétablir les droits fondamentaux, dans les cas où ils avaient été violés par eux-mêmes ou par un organe inférieur. Il s'agit là de décisions, qui plus est, contre lesquelles il n'est pas légalement possible pour le moment d'interjeter un recours aux fins de préserver et rétablir, par la voie judiciaire, le droit ou la liberté

66. Art. 3.11 de la loi 50/1981: «Para el cumplimiento de las misiones establecidas en el artículo 1, corresponde al Ministerio Fiscal: [...] 11. Intervenir en los procesos judiciales de amparo [...]».

67. Art. 3.3 de la loi 50/1981: "Para el cumplimiento de las misiones establecidas en el artículo 1, corresponde al Ministerio Fiscal: [...] 3. Velar por el respeto [...] de los derechos fundamentales y libertades públicas con cuantas actuaciones exija su defensa».

68. Art. 249.1 de la loi $1 / 2000$ de procédure civile.

69. Art. 114 sq. de la loi $2 / 1998$ du contentieux administratif.

70. Art. 116 de la loi organique 5/1985 du régime électoral général.

71. Art. 177 sq. de la loi $36 / 2011$ de juridiction sociale.

72. Art. 518 de la loi organique $2 / 1989$ sur le procès militaire.

73. Loi organique $4 / 1984$ sur la procédure d'habeas corpus. 
violés (et qui devraient être admis) ${ }^{74}$. Il en va de même pour la question de l'élargissement de l'objet du recours d'amparo, puisque l'argument avancé est en définitive celui du principe d'effectivité ou «d'effet utile» pour briser les règles.

Pour ce qui est du second principe, toujours en opposition avec l'opinion majoritaire qui veut qu'il y ait une atteinte "effective» au droit, il a été argumenté (également sur la base des articles 41.3, 49.1 et 54 de la LOTC) que le recours d'amparo possède le double objectif de "préserver», dans certains cas, et de "rétablir», dans d'autres, le droit. La thèse majoritaire a construit l'amparo autour du "rétablissement» du droit (caractère réparateur), mais n'a prêté que peu d'attention à la préservation des droits. Le rétablissement tardif n'est pas une protection. La préservation met préalablement à l'abri du dommage ou du danger ${ }^{75}$. Ce faisant, on défend la possibilité du recours d'amparo face à des «menaces» ou des lésions «futures" aux droits ${ }^{76}$, possibilité qui a existé dans les précédents médiévaux de l'amparo espagnol et que l'on peut observer aujourd'hui dans l'amparo mexicain.

Ces propositions sont risquées. Le Tribunal constitutionnel a été conçu par le constituant sur la base du principe d'une intervention extraordinaire ${ }^{77}$. Aller au-delà du caractère «subsidiaire " et «réparateur» de l'amparo aboutirait à une véritable mutation qui ferait passer le Tribunal constitutionnel d'acteur « extraordinaire» à intervenant « ordinaire».

\section{Le champ de protection de l'amparo constitutionnel}

Il existe une différence théorique entre l'amparo ordinaire et l'amparo constitutionnel qui ne s'est toutefois pas traduite dans la pratique. L'article 53.2 de la Constitution stipule:
Tout citoyen pourra demander la protection des libertés et des droits reconnus à l'article 14 et à la section première du chapitre deux ${ }^{78}[\ldots]$, le cas échéant, par le recours d'amparo devant le Tribunal constitutionnel ${ }^{79}$.

Par sa teneur, cette disposition semble laisser une certaine marge discrétionnaire («le cas échéant») tant dans l'établissement que dans la délimitation du champ de protection du recours, à la différence de ce qui se passe dans l'amparo ordinaire pour lequel tous les droits contenus dans ces articles doivent être protégés. Cependant, la LOTC a opté pour une position maximaliste en garantissant le recours d'amparo constitutionnel pour tous les droits pour lesquels la Constitution a laissé cette possibilité ouverte. Cette position est consacrée par l'article 41 de la LOTC aux termes duquel:

Les droits et libertés reconnues aux articles 14 à 29 de la Constitution sont susceptibles d'amparo constitutionnel

[...]. Cette même protection est applicable à l'objection de conscience reconnue à l'article 30 de la Constitution ${ }^{80}$.

Cela signifie que l'on ne peut se prévaloir du RAC que pour les droits constitutionnels expressément mentionnés dans ces dispositions; et qu'on ne peut l'introduire pour la protection d'autres droits visés soit dans d'autres dispositions de la Constitution, soit dans des traités internationaux, ou encore dans des lois internes.

Les données statistiques contenues dans les rapports d'activité du Tribunal constitutionnel ${ }^{81}$ font état de certaines tendances constantes. Le droit fondamental le plus invoqué est celui de l'article 24 de la Constitution («protection juridique effective par les cours et tribunaux») dont le contenu a été extraordinairement élargi par la jurisprudence constitutionnelle, devenant ainsi un véritable "macrodroit ${ }^{82}$. Le deuxième droit le plus invoqué est ensuite le droit à l'égalité devant la loi (art. 14 de la Constitution $)^{8_{3}}$. Les autres droits distincts des deux précédents n'ont pas été invoqués dans plus de $25 \%$ des affaires ${ }^{84}$.

74. Opinion dissidente du magistrat Manuel Jiménez de Parga y Cabrera (à laquelle se rallie le magistrat Vicente Gimeno Sendra) à la STC 63/1996 du 16 avril.

75. Opinion dissidente du magistrat Manuel Jiménez de Parga y Cabrera à la STC 27/1997 du 11 février.

76. C'est ainsi que l'envisage l'obiter dictum de Vicente Gimeno Sendra. Voir J. L. Cascajo Castro, V. Gimeno Sendra, El recurso de amparo, $2^{\mathrm{e}}$ éd., Madrid, Tecnos, 1988, p. 136 et 151

77. Voir R. L. Blanco Valdés, «La política y el derecho: veinte años de justicia constitucional y democracia en España (apuntes para un balance)», Teoría y realidad constitucional, $\mathrm{n}^{\circ}$ 4, 1999, p. 241-272, spéc. p. 249-251.

78. Art. 14-29 de la Constitution.

79. «Cualquier ciudadano podrá recabar la tutela de las libertades y derechos reconocidos en el artículo 14 y la Sección 1 del Capítulo Segundo [...], en su caso, a través del recurso de amparo ante el Tribunal Constitucional.» L'article ajoute que ce dernier recours «sera applicable à l'objection de conscience, reconnue à l'article 30 " ("será aplicable a la objeción de conciencia reconocida en el artículo 30 »), mais cette phrase a perdu son sens depuis l'abrogation du service militaire obligatoire en 2000.

80. «Los derechos y libertades reconocidos en los artículos catorce a veintinueve de la Constitución serán susceptibles de amparo constitucional [...]. Igual protección será aplicable a la objeción de conciencia reconocida en el artículo treinta de la Constitución.»

81. Ces données sont disponibles dans les rapports d'activité publiés par la Cour constitutionnelle depuis 1999, en ligne: https://www.tribunalconstitucional. es/es/memorias/Paginas/default.aspx.

82. Le pourcentage d'affaires dans lesquelles ce droit fut invoqué oscille entre un maximum de $89,22 \%$ (10 234 affaires) en 2006 et un minimum de $72,68 \%$ (5570 affaires) en 2014. Pour la dernière année pour laquelle les informations sont disponibles (2015), le pourcentage est de 75,19 \% (5 416 affaires).

83. Le pourcentage d'affaires dans lesquelles ce droit fut invoqué oscille entre un maximum de 23,66 \% ( 1600 affaires) en 2000 et un minimum de $13,77 \%$ (992 affaires) en 2015, dernière année pour laquelle les informations sont disponibles.

84. Un minimum de $18,63 \%$ (1357 affaires) en 2002 et un maximum de $25,69 \%$ (1851 affaires) en 2015 . Il faut noter que dans une même affaire peuvent être invoqués plusieurs droits fondamentaux. 
Le grand nombre d'affaires d'amparo auprès du Tribunal constitutionnel et le nombre important d'affaires découlant de l'article 24 de la Constitution ont soulevé un débat autour du champ de protection dudit recours.

Certaines propositions ont tout d'abord été formulées afin de restreindre le champ des droits protégés par le RAC, en se fondant sur la teneur littérale de l'article 53.2 de la Constitution ( «le cas échéant») qui semble considérer que les droits ayant vocation à être protégés par la voie de l'amparo ordinaire ou «judiciaire» n'ont pas tous à l'être également, et pas nécessairement, par le biais de l'amparo constitutionnel. Cette restriction a pour but de mettre un terme à l'avalanche de recours d'amparo qui inonde le Tribunal (notamment dans les années les plus critiques: 2005-2010). Concrètement, et en optant pour une solution plus radicale, il est ainsi proposé de priver du RAC les droits consacrés à l'article 24 de la Constitution ${ }^{85}$ (relatif à la protection juridique effective) et qui représentent le plus grand nombre de recours. Cette thèse a été rejetée par certains pour des raisons de politique constitutionnelle ${ }^{86}$ et par d'autres parce qu'ils la jugeaient inconstitutionnelle ${ }^{87}$.

Dans un sens tout à fait opposé, certains se sont interrogés sur le fait de savoir s'il ne conviendrait pas d'étendre le champ du recours d'amparo pour connaître des violations non seulement des droits constitutionnels visés à l'article 53.2 de la Constitution, mais également d'autres droits constitutionnels (tels que ceux visés au chapitre II du titre I) afin de le rapprocher de l'amparo de la Convention EDH et ainsi éviter que la Cour européenne des droits de l'homme ne condamne l'Espagne sans que le Tribunal constitutionnel ait pu se prononcer ${ }^{88}$. Cette proposition, en outre, permettrait de résoudre en partie le grave problème de l'encombrement de la Cour européenne des droits de l'homme. Si la restriction du champ de protection peut se fonder sur le libellé de l'article 53.2 de la Constitution, il n'y a cependant pas d'empêchement à l'élargissement dans la mesure où l'article 161.1.d de la Constitution laisse ouverte la possibilité d'attribuer de nouvelles compétences au Tribunal si une loi organique en dispose ainsi.

\section{Légitimation du recours d'amparo}

Conformément aux dispositions de l'article 46 de la LOTC, deux catégories de requérants sont fondés à introduire un recours d'amparo: les titulaires des droits fondamentaux concernés, ainsi que certains organes de l'État. Cette double légitimation est le reflet des dimensions subjective et objective des droits fondamentaux ${ }^{89}$.

D'abord, les droits fondamentaux sont des droits subjectifs et, en conséquence, les sujets titulaires des droits peuvent saisir le Tribunal pour défendre leurs prétentions. Les titulaires "typiques» sont les personnes physiques ou les personnes morales de droit privé, mais la reconnaissance de l'article 24 de la Constitution (accès à la justice) comme «droit fondamental» a ouvert la possibilité à des entités de droit public d'introduire des amparo pour la défense de leurs «droits et intérêts». De fait, les entités publiques ont introduit en 2015 quatre-vingt-douze amparo. Cet article n'est pas le lieu pour une discussion théorique sur la pertinence ou non de reconnaître les entités publiques comme titulaires de droits fondamentaux. Du point de vue classique, les entités publiques disposent de «compétences» plutôt que de "droits", mais le nœud du problème se trouve dans la qualification des règles procédurales comme «droits fondamentaux ». Une qualification plus adéquate à notre avis serait de les considérer comme des «garanties» des droits, et non comme des «droits» eux-mêmes. En fait, en droit espagnol (à la différence de ce qui se passe dans la Convention américaine des droits de l'homme) il n'existe pas de «droit à l'amparo» parce que l'amparo est placé dans le chapitre sur les "garanties» des droits et libertés. Si on avait considéré la protection judiciaire des droits (constitutionnels ou non) comme une "garantie» et non comme un «droit», le problème des prétendus «droits fondamentaux» des entités publiques ne se serait probablement pas posé.

Ensuite, les droits fondamentaux constituent également une partie importante du droit objectif. À ce titre, l'intérêt de leur protection ne relève pas seulement du titulaire du droit subjectif fondamental mais de toute la communauté. En conséquence, certains organes de l'État, en l'occurrence le Procureur (ministère public ou Ministerio Fiscal) et le Médiateur (défenseur du peuple ou Defensor del Pueblo) peuvent interjeter un recours d'amparo constitutionnel.

Le Médiateur (Defensor del Pueblo), en vertu de l'article 54 de la Constitution espagnole, est un «haut mandataire des Cortes Generales chargé [...] de défendre les droits [fondamentaux] ${ }^{90}$. Si, d'après l'article 29 de la loi organique 3/1981 sur le Médiateur, il peut introduire des requêtes d'amparo constitutionnel, ce dernier n'a introduit qu'une seule requête d'amparo, en 2015.

85. Voir P. Sala Sánchez, La delimitación de funciones entre las jurisdicciones constitucional y ordinaria en la protección de los derechos fundamentales, Madrid, Consejo general del poder Judicial, 1994, p. 35.

86. Voir F. Rubio Llorente, J. Jiménez Campo, Estudios sobre jurisdicción constitucional, Madrid, McGraw Hill, 1998, p. 59.

87. Voir G. Fernández Farreres, El recurso de amparo según la jurisprudencia del Tribunal constitucional, Madrid, Marcial Pons, 1994, p. 13; M. Aragón Reyes, «La reforma de la Ley Orgánica del Tribunal constitucional», Revista española de derecho constitucional, $\mathrm{n}^{\circ}$ 85, 2009 , p. 11 sq.

88. Voir la présentation par le président Pedro Cruz Villalón de la Memoria 1999 du Tribunal constitutionel, «Las tareas del Tribunal Constitucional», en ligne: http://www.tribunalconstitucional.es/es/memorias/Documents/Memoria\%201999.pdf.

89. Pour une réflexion approfondie sur la nature juridique et les dimensions des droits fondamentaux, voir C. Ruiz Miguel, Derechos fundamentales $y$ derecho procesal constitucional: transformación, evolución, transmutación, Mexico, Porrúa, 2014, p. 3-27.

90. "[...] alto comisionado de las Cortes Generales, designado [...] para la defensa de los derechos [fondamentales] [...].» 
Le Procureur (Ministerio Fiscal), selon l'article 124.1 de la Constitution espagnole, «a pour mission de promouvoir l'action de la justice en défense de la légalité, des droits des citoyens et de l'intérêt public protégé par la loi $"{ }^{91}$. D'après l'article 3.12 de la loi 50/1981 portant statut du Procureur, il est explicitement compétent pour introduire des requêtes d'amparo constitutionnel. De plus, l'article 47.2 de la LOTC prévoit que «le Procureur intervient dans tous les procès d'amparo, pour défendre la légalité, les droits des citoyens et l'intérêt public protégé par la loi ${ }^{92}$. En d'autres termes, il est toujours partie au procès même s'il n'a pas exercé le recours. Si le Procureur est partie prenante dans toutes les procédures d'amparo constitutionnel, il est cependant très rare de le voir introduire une requête d'amparo ${ }^{93}$.

De plus, l'article 47.1 de la LOTC stipule que peuvent comparaître dans un procès d'amparo constitutionnel, en qualité de défendeur ou d'intervenant, deux catégories de personnes: d'une part, les personnes favorisées par la décision, acte ou fait en raison duquel le recours est formulé (qui interviennent comme défenderesses), et, d'autre part, celles "ayant un intérêt légitime " quant à celui-ci (qui agissent en tant qu'intervenants auprès du demandeur ou du défendeur).

\section{L'objet de l'amparo}

L'objet de l'amparo constitutionnel est clair dans le texte de la loi, mais la jurisprudence constitutionnelle a en fait depuis longtemps une interprétation «créative» (voire illégale). De façon générale, l'objet du recours d'amparo est constitué des

[...] dispositions, actes juridiques, omissions ${ }^{94}$ ou simples voies de fait des pouvoirs publics de l'État, des communautés autonomes et des autres organismes publics à caractère territorial, corporatif ou institutionnel, ainsi que de leurs fonctionnaires ou agents ${ }^{95}$

qui violent les droits relevant du champ de protection de l'amparo.

Trois types de normes peuvent donner lieu à un recours à l'amparo, détaillés dans la LOTC: actes ou dispositions non législatives des Parlements (national ou régionaux) ${ }^{96}$ ou des pouvoir exécutifs (national ou régionaux $)^{97}$ et actes ou omissions des organes judiciaires (art. 44.1 de la LOTC). D’après les rapports d'activité du Tribunal constitutionnel, l'écrasante majorité des amparo concerne des résolutions judiciaires et seule une petite minorité est adressée contre des actes parlementaires. Il n'existe en pratique pas d'amparo directs contre des actes du gouvernement, ces actes ayant été au préalable contestés dans le contentieux administratif ${ }^{98}$.

On peut déduire de ce qui précède que, dans sa conception originale, l'objet du RAC ne peut être constitué que par des actes (normes particulières) provenant de l'un quelconque des pouvoirs publics (exécutif, législatif et judiciaire). Étaient ainsi initialement exclues du recours d'amparo plusieurs catégories normatives:

- tous les actes ou normes élaborés par les particuliers;

- les normes générales de nature réglementaire (qui ne pouvaient être attaquées que dans le cadre du contentieux administratif);

- les normes générales ayant rang de loi (qui ne pouvaient être contestées que par voie abstraite - recours et question d'inconstitutionnalité).

Cette conception initiale a subi certaines modifications. L'objet du recours d'amparo a été au centre d'une mutation légale, ainsi que d'un débat de doctrine et de jurisprudence. Comme nous avons eu l'occasion de l'exposer, l'intention première du RAC était d'exercer un contrôle des seuls actes ou normes particuliers émanant des pouvoirs publics. Son champ d'application s'est étendu dans plusieurs directions, sous l'impulsion de la doctrine et de la jurisprudence. Premièrement, le RAC a subi une mutation élargissant son objet aux actes émanant des particuliers. Deuxièmement, le RAC a été étendu, avec certaines restrictions, aux normes générales réglementaires. Et, troisièmement, on a voulu l'étendre, dans certaines conditions, aux normes générales législatives.

1) La première étape de l'élargissement de l'objet de l'amparo a été franchie avec une mutation légale permettant d'inclure les violations de droits fondamentaux du fait de particuliers. Dans un premier temps, le Tribunal constitutionnel, en accord avec le texte de la loi, a rejeté la possibilité du RAC dans les cas cités. C'est ainsi qu'une

91. "[...] tiene por misión promover la acción de la justicia en defensa de la legalidad, de los derechos de los ciudadanos y del interés público tutelado por la ley [...].»

92. "El Ministerio Fiscal intervendrá en todos los procesos de amparo, en defensa de la legalidad, de los derechos de los ciudadanos y del interés público tutelado por la Ley."

93. Entre 1999 (date à laquelle la Cour constitutionnelle commence à fournir des informations sur ce sujet) et 2015, le procureur a introduit treize requêtes d'amparo: 2015 (2), 2014 (4), 2013 (5), 2009-2012 (o), 2008 (1), 2001-2007 (o), 2000 (1), 1999 (o).

94. L'addition du mot «omisiones» fut faite dans la modification de la LOTC par la loi organique 6/2007 du 24 mai.

95. Art. 41.2 de la LOTC: "[...] disposiciones, actos jurídicos, omisiones o simple vía de hecho de los poderes públicos del Estado, las Comunidades Autónomas y demás entes públicos de carácter territorial, corporativo o institucional, así como de sus funcionarios o agentes».

96. Art. 42 de la LOTC: «Las decisiones o actos sin valor de Ley, emanados de las Cortes o de cualquiera de sus órganos, o de las Asambleas legislativas de las Comunidades Autónomas, o de sus órganos [...]».

97. Art. 43.1 de la LOTC: «disposiciones, actos jurídicos, omisiones o simple vía de hecho del Gobierno o de sus autoridades o funcionarios, o de los órganos ejecutivos colegiados de las comunidades autónomas o de sus autoridades o funcionarios o agentes [...]».

98. En 2015, il y a eu 13 amparo contre des résolutions parlementaires et 7190 contre des résolutions judiciaires; aucun amparo direct contre des actes du pouvoir exécutif. 
ordonnance motivée (auto) du Tribunal constitutionnel rappelle que

[...] pour réagir contre les violations présumées des droits fondamentaux ou libertés publiques qu'un particulier a commises dans la sphère juridique d'un autre particulier, ce dernier dispose, selon les cas, de la voie pénale ou de la voie civile, réglementées, concrètement, dans les articles 2 à 5 et 11 à 15 de la loi $62 / 1978^{99}$

pour conclure que le recours d'amparo devant le Tribunal constitutionnel (RAC), distinct de l'amparo ordinaire, " comme chacun sait, n'est recevable qu'à l'encontre d'actes des pouvoirs publics (art. 41.2 de la LOTC)». En fait, cet ATC 162/1982 ne faisait que rejeter la virtualité du RAC inter privatos.

Cette conception a été remise en cause peu de temps après par le Tribunal constitutionnel lui-même qui a établi une doctrine ouvrant le RAC aux violations des droits inter privatos en usant de la fiction (non explicitement) qui consiste à imputer au juge la méconnaissance du droit fondamental s'il ne reconnait pas sa violation par un particulier ${ }^{100}$. Le problème de cette construction de «l'assomption judiciaire» est que le juge n'a pas lésé le particulier dans son droit. En effet, il y a eu lésion avant intervention du juge; de plus, si le juge ne fait que confirmer un comportement privé qui a porté atteinte au droit fondamental, l'acte du juge n'est pas directement et immédiatement responsable du préjudice, comme l'exige la LOTC ${ }^{101}$.

2) L'idée que les normes générales à caractère réglementaire puissent aussi donner lieu au recours d'amparo constitutionnel a progressivement gagné du terrain.

Dans un premier temps, et de façon générale, le Tribunal a considéré que

L'objet et la fonction du recours d'amparo [...], sont, entre autres, des raisons qui mettent en évidence le fait que ces requêtes directes en contestation sont non recevables à l'encontre de dispositions générales et, dès lors, de dispositions réglementaires ${ }^{102}$.

Cependant, la jurisprudence a rapidement modifié ce critère en établissant une distinction entre deux types de règlements:

- d'une part, les règlements qui, même sans être formellement des normes générales, constituent maté- riellement des normes particulières, c'est-à-dire ceux que l'on appelle « règlements d'auto-application » ou «règlements particuliers », qui peuvent être contestés et annulés par le Tribunal par voie de $\mathrm{RAC}^{103}$;

- d'autre part, les règlements qui, non seulement portent le nom de cette catégorie, mais constituent également, en substance, des normes générales. Le traitement accordé par le Tribunal constitutionnel aux règlements de cette dernière catégorie a connu une évolution.

Initialement, le Tribunal admettait la possibilité de juger ces règlements par la voie du RAC, et, en cas de recevabilité du recours, seuls les actes d'application de ces règlements étaient annulés. Il laissait aux tribunaux du contentieux administratif toute compétence pour annuler les règlements ${ }^{104}$.

Le Tribunal a, par la suite, modifié sa doctrine quand en 1990 il a annulé plusieurs règlements généraux qui constituaient aussi des normes matériellement générales $^{105}$. La possibilité de jugement par voie d'amparo des règlements matériellement généraux semble ainsi consolidée ${ }^{106}$. Cette évolution a été saluée par la doctrine qui a jugé pertinent le changement de critère au nom de l'effectivité de l'amparo ${ }^{107}$, qui peut ainsi être considéré comme consolidé. Le problème, à notre sens, est qu'une telle solution, qui est guidée par le principe de l'effectivité (ce que la jurisprudence communautaire appellerait «l'effet utile»), bouleverse non seulement le principe de répartition des compétences entre la juridiction ordinaire et la juridiction constitutionnelle, mais aussi la nature même de la fonction judiciaire dans l'État constitutionnel européen continental.

3) Finalement, s'est posée la question de savoir si la contestation de normes générales à caractère législatif pouvait ou devait être possible par voie d'amparo. Il faut d'abord préciser qu'il n'y a pas amparo contre des lois en droit espagnol. Cela dit, à l'instar des règlements administratifs, il convient de distinguer ici les lois «matériellement » particulières et les lois "matériellement» générales. Le problème s'est posé dans toute son acuité lorsqu'en 1983 une importante loi particulière ${ }^{108}$ a été adoptée pour exproprier l'un des principaux groupes économiques espagnols sans appliquer les dispositions prévues par la loi (générale)

99. ATC $162 / 1982$ du 5 mai ( $1^{\text {re }}$ section).

100. STC $78 / 1982$ du 20 décembre $\left(\mathrm{FJ}^{\circ}\right.$ ).

101. Voir J. M. Bilbao Ubillos, La eficacia de los derechos fundamentales frente a particulares, Madrid, Centro de estudios políticos y constitucionales, 1997 , p. 168 .

102. STC $40 / 1982$ du 30 juin $\left(\mathrm{FJ}_{3}^{\circ}\right)$.

103. STC 9/1986 du 21 janvier. Cette construction rappelle l'article 263.4 du Traité sur le fonctionnement de l'Union européenne.

104. STC $162 / 1985$ du 29 novembre.

105. SSTC 7/1990 du 18 janvier; 32/1990 du 26 février; 61/1990 du 29 mars.

106. Cela apparaît, de façon nuancée (sans entrer dans la discussion de savoir si la décision annulée est une norme générale ou particulière), dans la STC 48/1998 et, plus clairement, dans la STC 40/1999 qui a admis un RAC contre un règlement matériellement général, bien qu’il ait été débouté pour des motifs de fond.

107. Voir E. García de Enterría, «La eliminación general de las normas reglamentarias nulas con ocasión de recursos contra sus actos de aplicación », Revista española de derecho administrativo, $\mathrm{n}^{\circ} 66,1990$, p. 279-294.

108. Loi $7 / 1983$ du 29 juin d'expropriation du groupe Rumasa. Pour mesurer l'importance de cette loi il faut rappeler que le groupe Rumasa était alors le groupe économique le plus important d'Espagne. 
d'expropriation forcée ${ }^{109}$. Cette loi a fait l'objet de plusieurs questions d'inconstitutionnalité posées par les juges ordinaires, toutes rejetées par le Tribunal constitutionnel ${ }^{110}$. Finalement, le principal propriétaire du groupe exproprié (M. Ruiz-Mateos) s'est adressé à la Cour européenne des droits de l'homme qui a statué en déclarant que l'Espagne et, plus précisément, le Tribunal constitutionnel, avait porté atteinte à son droit à un procès équitable (art. 6 de la Convention EDH) parce qu'après que plusieurs tribunaux ordinaires ${ }^{111}$ ont déclaré recevables les questions d'inconstitutionnalité de la loi (l'expropriation du groupe de M. Ruiz-Mateos), alors que la représentation légale de l'administration avait pu défendre la constitutionnalité de la loi ${ }^{112}, M$. Ruiz-Mateos n'avait pu être partie à ce procès ${ }^{113}$.

Dans l'éventualité d'une loi particulière, on avait formulé deux thèses : la première consisterait à admettre comme prémisse première le fait que les lois particulières sont conformes à la Constitution et à pallier le problème en permettant le recours à l'amparo à l'encontre de lois individuelles ${ }^{114}$; la deuxième possibilité, pour laquelle nous nous prononçons, consiste à rejeter la première prémisse, en considérant que les observations déjà présentées en son temps par Carl Schmitt sur ce point ${ }^{115}$ gardent toute leur valeur, ce qui permet donc de nier la constitutionnalité des lois particulières. Pour ce qui est des lois «matériellement» générales, il n'y a pas de cas de contestation directe de celles-ci. En tout état de cause, et indépendamment du fait qu'aucune décision du Tribunal n'ait annulé une loi dans le cadre d'une procédure d'amparo, nous avons estimé que l'argumentation du Tribunal en vue de juger dans le cadre du RAC les règlements administratifs est applicable en substance à cette autre norme générale appelée loi ${ }^{116}$.
Néanmoins, le législateur s'est penché sur une troisième voie qui, tout en acceptant la prémisse de la première thèse (c'est-à-dire la constitutionnalité des lois particulières), ne lui donne pas la même suite. On a ainsi ajouté en 2007 un nouvel alinéa à l'article 37 de la loi organique $\mathrm{du}$ Tribunal constitutionnel stipulant qu'une fois que la question de la constitutionnalité d'une loi posée par le juge ordinaire a été considérée recevable par le Tribunal constitutionnel, les personnes qui ont été parties dans la procédure judiciaire ordinaire peuvent elles aussi ester en justice devant le Tribunal constitutionnel pour défendre leurs arguments ${ }^{117}$.

\section{La modification de la recevabilité du recours d'amparo et la mutation de sa nature}

Les règles en matière de recevabilité du recours d'amparo, prévues à l'article 50 de la LOTC, ont été modifiées en 1988 (loi organique 6/1988 du 9 juin), puis en 2007 (loi organique $6 / 2007 \mathrm{du} 24 \mathrm{mai}$ ), pour tenter de freiner l'incessante augmentation des recours d'amparo déposés auprès du Tribunal. Même s'il est vrai que cette dernière réforme a réduit le nombre de recours qui peuvent parvenir au Tribunal, elle n'a pas opéré de réduction si significative qu'elle puisse justifier la mutation dont l'amparo a été objet.

Il existe deux conditions de recevabilité du recours d'amparo, l'une portant sur la forme, l'autre sur le fond.

Formellement, «[1]e recours doit satisfaire aux conditions figurant dans les articles 41 à 46 et $49{ }^{118}$. Néanmoins, tant la loi elle-même que la pratique du Tribunal font

109. La loi (générale) d'expropriation forcée pour cause d'utilité publique (encore en vigueur) est une loi du 16 décembre 1954

110. SSTC 166/1986 du 19 décembre; 6/1991 du 15 janvier.

111. Art. 163 de la Constitution: «Cuando un órgano judicial considere, en algún proceso, que una norma con rango de ley, aplicable al caso, de cuya validez dependa el fallo, pueda ser contraria a la Constitución, planteará la cuestión ante el Tribunal Constitucional en los supuestos, en la forma $y$ con los efectos que establezca la ley, que en ningún caso serán suspensivos» [ QQuand un organe judiciaire considère, au cours d'un procès, qu'une norme législative, applicable en l'espèce, de la validité de laquelle dépend le jugement, pourrait être contraire à la Constitution, il pose la question à la Cour constitutionnelle dans les conditions, dans la forme et avec les effets établis par la loi, qui en aucun cas ne sont suspensifs»].

112. Dans les procédures relatives à la constitutionnalité des lois, l'ancien article 37, alinéa 2 (aujourd'hui alinéa 3) de la loi organique du Tribunal constitutionnel dispose: «El Tribunal Constitucional dará traslado de la cuestión al Congreso de los Diputados y al Senado por conducto de sus Presidentes, al Fiscal General del Estado, al Gobierno, por conducto del Ministerio de Justicia, y, en caso de afectar a una Ley o a otra disposición normativa con fuerza de Ley dictadas por una Comunidad Autónoma, a los órganos legislativo y ejecutivo de la misma, todos los cuales podrán personarse y formular alegaciones sobre la cuestión planteada en el plazo común improrrogable de quince días. Concluido éste, el Tribunal dictará sentencia en el plazo de quince días, salvo que estime necesario, mediante resolución motivada, un plazo más amplio, que no podrá exceder de treinta días» [«Le Tribunal constitutionnel communiquera la question au Congrès des députés et au Sénat par l'intermédiaire de leurs présidents, au Procureur général de l'État, au gouvernement par l'intermédiaire du ministère de la Justice et, au cas où elle concernerait une loi ou une autre disposition normative ayant force de loi d'une Communauté autonome, aux organes législatif et exécutif de celle-ci; tous pourront comparaître et formuler des allégations à propos de la question posée dans un délai commun de quinze jours ne pouvant pas être prorogé. Une fois celui-ci expiré, le Tribunal rendra son arrêt dans un délai de quinze jours, sauf s'il estime nécessaire, moyennant une décision motivée, un délai plus long mais qui ne pourra pas excéder trente jours»].

113. Cour EDH, 23 juin 1993, Ruiz-Mateos c. Espagne, série A, nº 262.

114. Voir J. A. Montilla Martos, «Defensa judicial versus ley singular de intervención», Revista española de derecho constitucional, $\mathrm{n}^{\circ}$ 40, 1994, p. 291-321, spéc. p. 314. Un auteur important a, quant à lui, insisté sur le fait que la «règle» est que, là où il y a protection (amparo), il y a protection face aux lois, indépendamment de leur caractère particulier. Voir P. Cruz Villalón, «Sobre el amparo », in La curiosidad del jurista persa y otros estudios sobre la Constitución, Madrid, CEPC, 1999, p. 495 sq., spéc. p. 503.

115. C. Schmitt, Teoría de la Constitución [1 ${ }^{\text {re }}$ éd. allemande: 1928], F. Ayala (trad.), Madrid, Alianza Editorial, 1982, p. 162.

116. C. Ruiz Miguel, «L'amparo constitutionnel en Espagne: droit et politique», Les cahiers du Conseil constitutionnel, $\mathrm{n}^{\circ} 10,2001, \mathrm{p} .150$ sq., spéc. p. 156.

117. Art. 37 , al. 2 de la LOTC, après la modification introduite par la loi organique 6/2007 du 24 mai: "Publicada en el "Boletín Oficial del Estado" la admisión a trámite de la cuestión de inconstitucionalidad, quienes sean parte en el procedimiento judicial podrán personarse ante el Tribunal Constitucional dentro de los 15 días siguientes a su publicación, para formular alegaciones, en el plazo de otros 15 días».

118. Art. 50.1.a de la LOTC: «Que la demanda cumpla con lo dispuesto en los artículos 41 a 46 y 49 ». 
preuve de souplesse. Il est ainsi prévu que «dans les cas susceptibles d'être irrecevables », le Tribunal doit notifier au pétitionnaire les motifs d'irrecevabilité, afin que, dans un délai de dix jours, il puisse corriger les défauts observés (art. 49.4 et 50.4 de la LOTC).

Depuis la réforme de 2007, il existe une recevabilité, paradoxale, pour des motifs de fond. L'article 50.1.b de la LOTC exige en effet

[...] que le contenu du recours justifie une décision sur le fond par le Tribunal constitutionnel en raison de son importance constitutionnelle particulière qui aura été appréciée en raison de son intérêt pour l'interprétation de la Constitution, de son application ou de son efficacité générale pour la détermination du contenu et de la portée des droits fondamentaux ${ }^{119}$.

D'après un ancien magistrat du Tribunal constitutionnel, pourtant défenseur de la nouvelle régulation, l'amparo n'est plus un instrument de «tutelle» face à toute lésion subjective du droit, mais est désormais un instrument de "contrôle», c'est-à-dire un instrument supplémentaire pour le contrôle de la constitutionnalité des normes inférieures ${ }^{120}$. La réforme de 2007 a cherché à obtenir une réduction quantitative du nombre des amparo au prix d'une véritable mutation de la nature de l'amparo constitutionnel, qui n'est plus une procédure spéciale pour la défense des droits fondamentaux en tant que droits subjectifs, mais une procédure spéciale pour la défense desdits droits en tant que normes objectives. Mais, au grand dam des défenseurs de la réforme, l'écrasante majorité des affaires traitées par le Tribunal continuent à être des amparo $^{121}$. Si le sacrifice de l'amparo comme instrument de tutelle des droits fondamentaux en tant que droits subjectifs cherchait à ce que le Tribunal prête davantage d'attention aux procédures de contrôle de constitutionnalité ${ }^{122}$, on peut dire qu'il a échoué puisque que le nombre d'arrêts en matière de contrôle de constitutionnalité n'a pas augmenté et que le respect des délais ne s'est pas substantiellement amélioré ${ }^{123}$.

Le Tribunal constitutionnel avait déterminé qu'il appartenait au requérant de justifier cette «importance constitutionnelle particulière $»^{124}$. Cependant, afin de renforcer la sécurité juridique, il s'est senti obligé de combler le vide de la loi ou au moins d'éclaircir les mentions les plus vagues. Dans un important arrêt de 2009, le Tribunal a énuméré, de façon systématique, mais non exhaustive, les cas qui présentent cette «importance constitutionnelle particulière» :
Un recours d'amparo satisfait à la condition d'importance constitutionnelle spéciale:

a) lorsqu'il soulève un problème ou révèle un aspect d'un droit fondamental susceptible d'amparo sur lequel il n'existe pas de jurisprudence du Tribunal constitutionnel, cas de figure déjà envisagé dans la STC 70/2009 du 23 mars 2009;

b) lorsqu'il donne au Tribunal constitutionnel l'occasion de clarifier ou de modifier sa jurisprudence au terme d'une réflexion menée en son sein - comme en l'espèce - ou en raison de nouvelles réalités sociales ou de modifications légales importantes pour la définition du contenu d'un droit fondamental, ou d'un changement de doctrine des organes chargés de l'interprétation des traités et accords internationaux visés à l'article $10, \$ 2$ de la Constitution;

c) lorsque la violation alléguée d'un droit fondamental trouve son origine dans la loi ou dans une autre disposition à caractère général;

d) lorsque la violation du droit fondamental est fondée sur une interprétation jurisprudentielle réitérée de la loi que le Tribunal constitutionnel considère comme attentatoire au droit fondamental et qu'il estime nécessaire d'en donner une autre interprétation conforme à la Constitution;

e) lorsque, de manière générale et réitérée, les juridictions ordinaires ne respectent pas la jurisprudence du Tribunal constitutionnel sur le droit fondamental en cause, ou lorsqu'il existe des décisions judiciaires revêtant un caractère contradictoire en ce qu'elles n'interprètent pas de la même manière la jurisprudence constitutionnelle sur le droit fondamental ou qu'elles l'appliquent dans certaines affaires mais pas dans d'autres;

f) lorsqu'un organe judiciaire refuse manifestement de suivre la jurisprudence constitutionnelle [article 5 de la loi organique relative au pouvoir judiciaire, RCL 1985 , 1578, 2635)];

g) enfin, lorsque l'affaire objet du recours, bien que ne relevant pas des hypothèses exposées ci-dessus, possède une portée qui dépasse le cas d'espèce en ce qu'elle pose une question juridique ayant des répercussions sociales ou économiques importantes, ou des conséquences politiques générales, ce qui pourrait être le cas, principalement mais pas exclusivement, de certains amparo électoraux ou parlementaires ${ }^{125}$.

La décision sur la recevabilité du recours d'amparo relève en principe des «sections» du Tribunal constitutionnel, qui sont au nombre de quatre (chacune étant composée de trois magistrats; art. 8 de la LOTC). Mais ce sont parfois les «chambres» (deux chambres, chacune de six magistrats) qui en décident. Les chambres sont appelées à se prononcer dans deux situations:

119. "Que el contenido del recurso justifique una decisión sobre el fondo por parte del Tribunal Constitucional en razón de su especial trascendencia constitucional, que se apreciará atendiendo a su importancia para la interpretación de la Constitución, para su aplicación o para su general eficacia, y para la determinación del contenido y alcance de los derechos fundamentales.»

120. Voir M. Aragón Reyes, «La reforma...», p. 40.

121. En 2015, le nombre des amparo s'élève à 7203 pour un total de 7369 affaires, soit $97,75 \%$ des affaires.

122. Voir M. Aragón Reyes, «La reforma... », p. 42.

123. Par exemple, le 2 février 2017 la cour a rendu plusieurs arrêts très significatifs après plus de trois ans d'attente dans des procédures de différentes natures: l'arrêt STC 15/2017 décide un recours d'inconstitutionnalité contre une loi introduite le 21 février 2013 (soit quatre ans auparavant); l'arrêt STC 16/2017 tranche un conflit de compétences introduit le 20 décembre 2012 (soit quatre ans auparavant); l'arrêt STC 17/2017 statue sur un amparo introduit le 26 février 2014 (soit trois ans auparavant).

124. SSTC $188 / 2008$ du 21 juillet; $289 / 2008$ et $290 / 2008$ du 22 septembre.

125. STC $155 / 2009$ du 25 juin. 
- si une section considère par deux voix contre une que l'affaire doit être examinée, la décision est déférée à la chambre. Si bien entendu la section considère que l'affaire n'est pas recevable par deux voix contre une la décision de la section s'impose;

- si le Procureur fait appel contre l'ordonnance de nonlieu de l'affaire.

En principe, la décision de recevabilité revêt la forme d'une ordonnance non motivée (providencia). Conformément aux articles 370 de la loi de procédure civile et $\mathbf{2 4 8 . 1}$ de la loi organique du pouvoir judiciaire (subsidiaires, à cet égard, aux termes de l'article 8o de la LOTC), la formule des ordonnances «se limite à mentionner le juge ou le tribunal, sans autres motivations juridiques ni ajouts que la date de son émission et le juge ou la chambre qui l'a prise», bien qu' «elles peuvent être succinctement motivées sans condition de forme particulière, en tant que de besoin ». Dans la pratique, le Tribunal constitutionnel a pour habitude d'inclure une motivation, même succincte.

Mais parfois les décisions revêtent la forme d' "ordonnances motivées» (autos), qui sont «toujours fondées» et contiennent «les faits et les raisonnements juridiques» (art. 248.2 de la loi organique du pouvoir judiciaire).

Les décisions du Tribunal sur la recevabilité d'une affaire ne peuvent être contestées ni par la partie qui se considère victime d'une violation des droits de l'homme, ni par le Médiateur (s'il est partie au procès). Seul le Procureur peut faire appel des décisions de recevabilité. Le recours du Procureur contre une décision de recevabilité est réglé par une ordonnance motivée du même organe qui a prononcé la décision d'irrecevabilité (recurso de súplica). Quant aux ordonnances motivées de rejet (autos), elles ne peuvent faire l'objet d'un appel (art. 50.4 de la LOTC).

Les ordonnances non motivées de rejet (providencias) ne sont pas publiées et les ordonnances motivées de rejet (autos) font l'objet d'une publicité restreinte: elles n'apparaissent pas au Boletín Oficial del Estado (Journal officiel) (où sont publiés tous les jugements du Tribunal constitutionnel), mais dans la collection officielle de la jurisprudence constitutionnelle publiée par le Tribunal. Les ordonnances motivées (autos) constituent une «jurisprudence mineure», mais prendre connaissance des arguments qui y sont développés par le Tribunal peut parfois présenter un grand intérêt.

Le nombre de décisions de non-lieu d'amparo constitutionnel a considérablement augmenté après la réforme de 2007 qui a durci les conditions de recevabilité du recours. Le nombre des décisions recevables s'est réduit de 4,75\% en 1999 à 1,09\% en 2015. Le nombre des affaires déclarées non recevables par ordonnance non motivée est important ${ }^{126}$.
Le régime de recevabilité du RAC a fait l'objet d'un débat doctrinal très intense avant la réforme de 2007 qui, au moins du point de vue de lege lata, semble être clos par l'arrêt de la Cour européenne des droits de l'homme dans l'affaire Arribas Anton c. Espagne. Les deux positions fondamentales du débat opposent un système de recevabilité réglé (que l'on peut dénommer «européen») et un système de recevabilité non réglé (que l’on peut appeler «américain»).

Toutes les formules employées dans les différentes versions de la loi organique du Tribunal constitutionnel se sont placées dans le système de recevabilité réglé, même si l'amparo s'est transformé en un instrument de dépuration des normes de droit objectif. Mais la question était de voir s'il était possible d'introduire en Espagne un système non réglé de recevabilité, comme celui des États-Unis (writ of certiorari), qui permettrait au Tribunal constitutionnel d'avoir un pouvoir discrétionnaire de décision en matière de recevabilité des recours, avec pour corollaire la possibilité de contrôler le volume des affaires qu'il peut traiter (docket control). La discussion se pose de lege lata et de lege ferenda.

De lege lata, on a opposé qu'il n'est pas possible d'introduire en Espagne le système du certiorari parce qu'il n'est pas adapté à la nature du recours d'amparo dans la mesure où il est configuré, d'une part, comme un droit de réaction et, d'autre part, comme un droit d'accès à une juridiction qui requiert, tant pour admettre ou rejeter un recours que pour y faire droit ou le débouter, une décision fondée en droit $^{127}$.

Mais il y a plusieurs raisons pour ne pas partager cet avis ${ }^{128}$. D'abord, le recours d'amparo n'est pas un «droit de réaction » car il n'y a pas de droit « inconditionnel» de recours devant le Tribunal constitutionnel (à la différence du recours prévu par la Convention américaine des droits de l'homme). Il est vrai que, lorsque la loi le permet, il suffit d'invoquer un intérêt légitime pour pouvoir exercer l'amparo; néanmoins, la Constitution n'octroie pas un «droit» à l'amparo (la Constitution, dans son article 53, alinéa 2, stipule que l'amparo constitutionnel existe «le cas échéant») et la loi ne précise pas quand il y a lieu d'accorder l'amparo. Ensuite, il a été également objecté que le principe de la décision motivée est une exigence du droit à la motivation des arrêts que le Tribunal constitutionnel lui-même reprend du droit à la protection judiciaire visé à l'article 24 de la Constitution. Mais ce droit a pour destinataires les juges et les tribunaux qui sont parties intégrantes du pouvoir judiciaire (juges ordinaires), et non le Tribunal constitutionnel (qui ne fait pas partie du pouvoir judiciaire), en plus du fait que le système connaît des décisions judiciaires qui ne requièrent pas

126. En 2012, 7292 affaires ont été déclarées irrecevables par «providencia» et seulement 2 déclarées irrecevables par «auto», mais en 1999 les chiffres respectifs étaient de 4369 contre 116.

127. Voir M. Sánchez Morón, El recurso de amparo constitucional: naturaleza jurídica, características actuales y crisis, Madrid, Centro de estudios constitucionales, 1987 , p. 30.

128. Voir C. Ruiz Miguel, «L'amparo constitutionnel en Espagne... », p. 157. 
de motivation (les providencias) et qu'à l'heure actuelle il est possible de rejeter une affaire par une telle ordonnance non motivée. Troisièmement, on peut objecter que le droit à la protection juridique est déjà satisfait par la décision motivée prise par les tribunaux ordinaires, le RAC étant une garantie extraordinaire.

La discussion semble avoir été close par la Cour européenne des droits de l'homme lorsqu'elle a statué sur la conformité de la nouvelle régulation de la recevabilité de l'amparo avec la Convention EDH. La Cour de Strasbourg avalise l'opinion déjà soutenue par Sánchez Morón que nous ne partageons pas pour les raisons qui viennent d'être exposées. La Cour a jugé que le «droit à un tribunal», dont le droit d'accès constitue un aspect, n'est pas absolu et qu'il se prête à des limitations implicitement admises, notamment en ce qui concerne les conditions de recevabilité d'un recours; néanmoins, les limitations appliquées ne doivent pas restreindre l'accès ouvert à l'individu d'une manière ou à un point tels que ce droit s'en trouve atteint dans sa substance même. En outre, elles ne s'accordent avec l'article $6, \$ 1$ de la Convention que si elles poursuivent un but légitime et s'il existe un rapport raisonnable de proportionnalité entre les moyens employés et le but visé $^{129}$. Les juges strasbourgeois ont assimilé l'amparo à une cassation $^{130}$ et ont rappelé que l'article 6 de la Convention n'astreint pas les États contractants à créer des juridictions d'appel ou de cassation et, encore moins, des juridictions compétentes en matière d'amparo. Néanmoins, un État qui se dote de juridictions de cette nature a l'obligation de veiller à ce que les justiciables jouissent auprès d'elles des garanties fondamentales de l'article 6 de la Convention. La Cour a conclu que l'application par les juridictions internes de formalités à respecter pour former un recours est susceptible de violer le droit d'accès à un tribunal ${ }^{131}$.

De lege ferenda, on peut se demander si un tel système non réglé de recevabilité serait convenable. À notre avis il n'y a point de doute à cet égard pour deux raisons. Premièrement, le système «réglé » ne reflète pas la réalité pratique de la procédure de recevabilité. Deuxièmement, ce modèle provoque des espoirs chez les citoyens qui sont massivement déçus avec un coût politique et constitutionnel très haut.

En premier lieu, il est temps de dévoiler au grand jour le fait que les prévisions légales sur la procédure de recevabilité ne correspondent pas à la réalité. Prenons le cas du Tribunal constitutionnel espagnol, composé de douze membres. Si nous faisons le calcul des jours de travail des magistrats, hors jours fériés, actes protocolaires et jours dédiés à la rédaction et la délibération des arrêts dans les procédures de contrôle de constitutionnalité des lois ou de conflits de compétences, le nombre de jours disponibles pour l'examen des amparo est très faible. Personne ne peut raisonnablement croire que les magistrats du Tribunal étudient eux-mêmes les 7203 amparo qui ont été portés devant le Tribunal en 2015 et rédigent les décisions de recevabilité (providencias). Il est tout à fait clair que, dans leur écrasante majorité, les affaires ont été examinées et les décisions rédigées non par les magistrats, mais par les conseillers (letrados) qui « assistent» (art. 97.1 de la LOTC) le Tribunal, composé des magistrats (art. 5 de la LOTC). En réalité, les conseillers n'assistent pas les magistrats... mais les remplacent dans cette tâche. Cette substitution (un secret de Polichinelle) a pour effet une perte de qualité technique et constitutionnelle de la décision, parce que même si les conseillers (letrados) sont qualifiés, la présomption iuris tantum est que les magistrats le sont davantage, ce qui les autorise à contrôler les décisions des organes judiciaires (y compris celles du plus élevé dans la structure judiciaire, le Tribunal suprême - la Cour de cassation). Ainsi, la prévision légale qui veut que les décisions importantes soient prises par les magistrats est démentie par la pratique: au vu du volume considérable d'affaires, la recevabilité est décidée, dans l'immense majorité des cas, par les seuls conseillers et non par les magistrats. On peut même penser que cette «assistance» qui est devenue «substitution » n'existe pas seulement pour l'étude des affaires et la rédaction des décisions de recevabilité, mais aussi dans la rédaction des projets d'arrêts d'amparo ${ }^{132}$. Dans tous les cas, même si nous acceptons que les conseillers préparent seulement, voire «assistent», la dissociation entre la préparation extra-juridictionnelle et la décision juridictionnelle peut être poussée très loin, mais on ne saurait cacher le fait que toute préparation à effet sélectif relève fonctionnellement d'un abandon du principe selon lequel la juridiction constitutionnelle est l'œuvre d'un tribunal constitutionnel compris comme un organe composé d'un nombre réduit de juges ${ }^{133}$.

Deuxièmement, la procédure de recevabilité retire au citoyen son espoir légitime qui veut qu'une fois remplies les exigences contenues dans la loi, le fond de son affaire sera examiné. Mais en raison du volume considérable d'affaires parvenues au tribunal, il est matériellement impossible pour les douze magistrats de faire un tel examen. Rappelons qu'en 2015, 98,91 \% des requêtes d'amparo ont été déclarées irrecevables. Personne ne peut douter qu'il existe dans ce total des requêtes dénuées de fondement ou pauvrement fondées... mais peut-on vraiment

129. Cour EDH, 20 janvier 2015, Arribas Antón c. Espagne, $\$ 41$.

130. Ibid., \$ 43 .

131. Ibid., $\$ 42$.

132. Voir P. S. Coderch, "Cinco propuestas para el Tribunal constitucional», El País, 12 juillet 2012. Il déclare explicitement: «[...] los magistrados habrán de esforzarse un poco más en escribir más partes de sus sentencias de propia mano y en redactarlas todas del modo más sencillo posible. Hay demasiadas sentencias de letrado, es decir, muy sustancialmente redactadas por los buenos letrados del Tribunal Constitucional, sin cuya ayuda ningún Tribunal de esta naturaleza puede funcionar. Mas la redacción de las sentencias es responsabilidad primaria de juez, no de letrado".

133. Voir O. Pfersmann, «Le recours direct: entre protection juridique et constitutionnalité objective», Les cahiers du Conseil constitutionnel, n 10 , 2001, p. 110 sq., spéc. p. 117. 
croire que seules 4,75\% des affaires ont rempli les exigences de recevabilité ? Ceci est difficile à croire. La conclusion qui en découle est claire: dans un nombre important de cas, le citoyen est déçu par le Tribunal constitutionnel qui déclare sa requête irrecevable même si elle semble remplir les exigences établies pour être acceptée et même permettre de gagner.

À notre avis, ces deux objections peuvent être surmontées en prenant en compte les caractéristiques du système de recevabilité. En premier lieu, le fait que le citoyen sache dès le début que la recevabilité relève d'une décision volontaire du Tribunal empêche la création d'attentes qui pourront être déçues. Bien au contraire, la logique étant de voir sa requête rejetée par le Tribunal suprême, personne ne peut en conséquence affirmer que son attente a été déçue. En second lieu, et à nouveau parce que la décision de recevabilité relève d'une libre décision du Tribunal et ne nécessite aucune justification, le coût de rédaction d'une telle décision est dérisoire. Deux mots seulement suffisent (certiorari denied) ou un simple ordre de dévolution de l'affaire à la juridiction d'où provient l'affaire pour qu'elle reconsidère sa résolution à la lumière de la doctrine du Tribunal suprême. Une prise de décision aussi expéditive permet deux choses importantes: que les magistrats eux-mêmes, et non d'autres personnes, puissent prendre les décisions d'irrecevabilité; que les magistrats aient davantage de temps à consacrer à l'étude approfondie des affaires déclarées recevables et à la rédaction personnelle des arrêts. On peut rappeler avec Otto Pfersmann qu' «aucune nécessité conceptuelle ne commande l'attribution de tous ces contrôles à une instance juridictionnelle unique ${ }^{134}$, de sorte que l'État de droit est préservé s'il a été porté connaissance d'une possible violation de ces droits fondamentaux par le tribunal.

\section{La procédure et l'arrêt du recours d'amparo}

Le recours peut être jugé par une section, une chambre ou l'assemblée plénière du Tribunal constitutionnel. La règle générale veut qu'une fois que le recours d'amparo a été jugé recevable il est déféré à l'une des deux chambres (de six membres chacune) qui composent le Tribunal (art. 48 de la LOTC); mais si le cas nécessite seulement l'application d'une jurisprudence bien connue du Tribunal, il relève de la section (art. 52.2 de la LOTC). Le cas peut enfin être réglé par l'assemblée plénière si la chambre veut s'écarter de sa jurisprudence habituelle:

Lorsqu'une chambre estime nécessaire de s'écarter, sur un point quelconque, de la jurisprudence constitutionnelle établie précédemment par le Tribunal, la question est soumise à la décision de son assemblée plénière ${ }^{135}$.

La singularité la plus marquante de l'amparo constitutionnel en Espagne est le caractère écrit de sa procédure. Une fois l'amparo déclaré recevable, le Tribunal transmet la requête aux autres parties, étant entendu que, comme souligné supra, le Procureur doit être toujours partie au procès d'amparo. À ce moment-là, il appartient au Tribunal constitutionnel de choisir entre demander aux autres parties de présenter un exposé écrit ou convoquer une audience publique pour que toutes les parties présentent oralement leur exposé. Les données des rapports d'activité du Tribunal nous offrent des informations tout à fait étonnantes: on compte ainsi seulement cinq affaires entendues oralement en vingt-quatre ans ${ }^{136}$. C'est une caractéristique marquante, et plutôt négative, du système espagnol. Un des effets négatifs de ce système est de nourrir le vice déjà dénoncé de la «substitution» des magistrats par les conseillers du Tribunal constitutionnel. En revanche, une audience publique assure que tous les magistrats aient pu connaître l'ensemble des positions des plaideurs. En outre, avec une audience publique, le problème des arrêts rendus en retard serait atténué, parce qu'il serait difficile de concevoir qu'ils soient rendus plusieurs années après l'audience publique.

Au cours de l'examen de l'affaire, la chambre (ou, le cas échéant, la section ou l'assemblée plénière) peut d'office ou sur la demande du pétitionnaire «suspendre l'exécution de l'acte des pouvoirs publics à propos duquel l'amparo constitutionnel est réclamé $»^{137}$. La possibilité de suspendre les décisions attaquées en amparo est à l'origine d'un des problèmes les plus graves qui se pose dans la pratique. Il devient de plus en plus habituel (et ceci explique le pourcentage élevé d'affaires pénales qui font l'objet d'un recours d'amparo) que les parties plaidantes qui ont perdu un procès face à la justice ordinaire recourent au Tribunal constitutionnel pour tenter d'éviter l'exécution de la décision de condamnation. Une fois qu'ils ont introduit le recours et dans l'attente de la décision de recevabilité rendue par le Tribunal constitutionnel, ils s'adressent au juge ordinaire à qui incombe l'exécution du jugement pour lui demander de suspendre celle-ci en arguant qu'un recours d'amparo a été formé et qu'il est pendant. Bien que formellement le cas ne présente pas de difficulté (seul le Tribunal constitutionnel peut ordonner cette suspension), cette pratique conduit, d'une part, à surcharger de recours le Tribunal constitutionnel et, d'autre part, à ce que certains tribunaux ordinaires prononcent la suspension dans de tels cas. Cependant, le nombre de mesures provisoires ou conservatoires (medidas cautelares) adoptées par le Tribunal (art. 56 de la LOTC) est très faible. D’après le

134. Ibid., p. 113

135. Art. 13 de la LOTC: «Cuando una Sala considere necesario apartarse en cualquier punto de la doctrina constitucional precedente sentada por el Tribunal, la cuestión se someterá a la decisión del Pleno».

136. Affaires entendues oralement: 2 en 1980-1986; pas de données pour 1987-1998; o en 1999-2000; 2 en 2001; 1 en 2002; o en 2003-2015. La dernière application d'une procédure orale date donc de 2002.

137. Art. 56.1 de la LOTC: «suspenderá [...] la ejecución del acto de los poderes públicos por razón del cual se reclame el amparo constitucional». 
rapport d'activité de 2015, période au cours de laquelle ont été déposées 7203 affaires d'amparo, le Tribunal constitutionnel avait prononcé seulement 23 ordonnances sur mesures provisoires... et seulement 6 avaient octroyé la mesure demandée.

Il est statué sur l'affaire par "arrêt» (sentencia) d'octroi ou de refus de l'amparo (art. 53 de la LOTC). L'arrêt d'octroi de l'amparo peut contenir "une ou plusieurs» des mentions suivantes: $1^{\circ}$ "reconnaissance du droit ou de la liberté publique, conformément à son contenu constitutionnellement déclaré ; $2^{\circ}$ " déclaration de nullité de la décision, acte ou résolution ayant empêché le plein exercice des droits ou libertés protégés, en déterminant, le cas échéant, l'étendue de ses effets»; et $3^{\circ}$ "rétablissement du requérant dans l'intégrité de son droit ou liberté et adoption, le cas échéant, des mesures propres à son maintien $»^{138}$.

La loi, dans sa première version de 1979, fixait un délai de «dix jours », « une fois les allégations [des parties] présentées ${ }^{139}$, délai qui n'a jamais été respecté. En pratique, le Tribunal avait l'habitude de prendre plusieurs années pour statuer après la présentation des allégations. Cette situation était tout à fait scandaleuse, ce qui explique la modification de la teneur de la disposition dans la réforme de 2007 prévoyant que le Tribunal statuera «dix jours» après la date qu'il aura lui-même déterminée ${ }^{140}$.

Dans la pratique habituelle, cependant, les arrêts d'amparo constitutionnel ne peuvent avoir que deux effets: déclaratoire ou d'annulation. Ce n'est qu'à titre exceptionnel que le Tribunal donne à ses arrêts un troisième effet, l'effet réparateur ( «rétablissement du requérant dans l'intégrité de son droit ou liberté et adoption, le cas échéant, des mesures propres à son maintien »), prévu à l'article 55.1.c de la LOTC.

Le Tribunal constitutionnel est très réticent à utiliser cette possibilité parce que cela peut provoquer, comme cela a déjà été le cas, des affrontements avec les tribunaux ordinaires, notamment avec le Tribunal suprême. Parmi les nombreux cas où un tel affrontement s'est produit, on peut citer l'affaire Preysler qui, par son intérêt juridique, mérite d'être rappelée. $\mathrm{M}^{\mathrm{me}}$ Isabel Preysler avait poursuivi un magazine qui avait publié un rapport qu'elle considérait préjudiciable à son droit à la vie privée. Dans son arrêt du 23 mai 1991, le tribunal de première instance $n^{\circ} 32$ de Barcelone a partiellement accueilli la demande et a condamné le magazine à payer un montant de 5 millions de pesetas à la victime. Les deux parties ont fait appel du jugement et, en appel, l'arrêt du 12 janvier 1993 de la onzième section de la cour provinciale de Barcelone a publié une déclaration dans laquelle elle a condamné le magazine à payer un montant du double de celui fixé en première instance (10 millions de pesetas). Le magazine condamné porta l'affaire en cassation, et le 31 décembre 1996 la première chambre du Tribunal suprême cassa l'arrêt précédent et rejeta la réclamation de $\mathrm{M}^{\text {me }}$ Preysler. Celle-ci avait alors formé contre le jugement une requête d'amparo sur laquelle il a été statué trois ans et demi plus tard, le 5 mai 2000 , avec une décision équivoque dans laquelle il était déclaré textuellement que l'amparo était accordé et où le Tribunal disait:

1. Reconnaître que l'on a porté atteinte au droit à la vie privée et familiale de la requérante.

2. Rétablir son droit et, à cet égard, annuler l'arrêt $\mathrm{n}^{\circ}$ 157/1996, publié le 31 décembre 1996 par la première chambre du Tribunal suprême dans l'appel $n^{\circ} 872 / 93^{141}$.

Le magazine a ensuite déposé un écrit avec les allégations de la partie auprès du Tribunal suprême demandant qu'il prononce une nouvelle peine. La première chambre du Tribunal suprême a alors rendu un nouvel arrêt, en date du 20 juillet 2000 , condamnant le magazine, mais à payer la somme dérisoire de 25000 pesetas. Face à cette décision du Tribunal suprême, $M^{\text {me }}$ Preysler a déposé un nouvel amparo constitutionnel au motif qu'une telle compensation équivalait à accepter la violation de sa vie privée et constituait même, à cet égard, une nouvelle violation de son droit à la vie privée. D'autre part, et pour éviter de nouvelles surprises liées à la procédure, le défendeur a demandé au Tribunal constitutionnel de préciser les effets de son arrêt. C'est ainsi que, dans un nouvel arrêt, le Tribunal constitutionnel a apporté quelques précisions relatives à ce troisième effet des arrêts de protection constitutionnelle ${ }^{142}$. Le Tribunal constitutionnel a ainsi décidé de reconnaître la force de chose jugée au quantum fixé dans l'arrêt de la cour provinciale (l'arrêt qui avait accordé le maximum). Cette décision a entraîné une gêne perceptible au sein du Tribunal suprême qui a abouti à plusieurs affrontements. Cela peut peut-être expliquer la réticence du Tribunal constitutionnel à faire pleinement usage des pouvoirs conférés par l'article 55.1.c de la LOTC.

138. Art. 55.1 de la LOTC (nous soulignons): «Reconocimiento del derecho o libertad pública, de conformidad con su contenido constitucionalmente declarado"; "Declaración de nulidad de la decisión, acto o resolución que hayan impedido el pleno ejercicio de los derechos o libertades protegidos, con determinación, en su caso, de la extensión de sus efectos"; "Restablecimiento del recurrente en la integridad de su derecho o libertad con la adopción de las medidas apropiadas, en su caso, para su conservación».

139. Première version de l'art. 52, al. 3: «Presentadas las alegaciones o transcurrido el plazo otorgado sin otros trámites, la Sala pronunciará la sentencia que proceda en el plazo de diez días» [ "Une fois les allégations présentées ou le délai accordé passé sans autres formalités, la chambre rendra son arrêt dans un délai de dix jours»].

140. Nouvelle rédaction après la loi organique 6/2007: «La Sala, o en su caso la Sección, pronunciará la sentencia que proceda en el plazo de 10 días a partir del día señalado para la vista o deliberación» [ «La chambre, ou le cas échéant la section, rendra l'arrêt dans un délai de 10 jours à compter de la date fixée pour l'audience ou la délibération "]. Par exemple, dans l'arrêt STC 17/2017, la dernière des allégations écrites fut présentée le 22 juin 2015, mais la Cour fixa la date de délibération au 2 février 2017.

141. STC $115 / 2000$ du 5 mai.

142. STC $186 / 2001$ du 17 septembre (FJ 9). 


\section{Le recours d'amparo dans la politique constitutionnelle}

Le grand débat qui sous-tend presque toutes les questions relatives au RAC n'est autre que celui de la relation entre le Tribunal constitutionnel et la justice ordinaire, relation qui s'est progressivement tendue. Et sur ce point, en effet, il y a accord quant aux causes de cette tension: à savoir, l'inclusion dans le champ de l'amparo des droits de procédure (art. 24 de la Constitution) et du principe d'égalité (art. 14 de la Constitution) et l'interprétation extensive qu'en a donnée le Tribunal constitutionnel. Alors même que la nature subsidiaire de l'amparo conduisait déjà le Tribunal constitutionnel à agir d'un certain point de vue comme une Cour de cassation, les recours fondés sur les articles 24 et 14 ont fait de lui une authentique Cour de cassation en lui permettant d'occuper le champ matériel que la Constitution réservé au pouvoir judiciaire ${ }^{143}$. La tension a atteint des niveaux critiques en 1994, 2000 et surtout 2004. En 1994, la chambre civile du Tribunal suprême a décidé à l'unanimité d'évoquer auprès du roi, en sa qualité de "pouvoir modérateur» (art. 56.1 de la Constitution), sa préoccupation et son malaise face à ce qu'elle considérait être « une invasion de la fonction juridictionnelle qui, conformément à la Constitution, revient aux juges et aux tribunaux» ${ }^{144}$. Le 15 décembre 2000, la chambre pénale du Tribunal suprême a adopté à l'unanimité une décision ${ }^{145}$ qui va assurément à l'encontre des pouvoirs que l'article 55.1.c de la LOTC confère au Tribunal constitutionnel. En 2003, la première chambre (civile) du Tribunal suprême avait accepté de juger la pertinence d'exiger responsabilité civile aux membres du Tribunal constitutionnel, même si dans son arrêt du 23 janvier 2004, le Tribunal suprême débouta le requérant.

Tout ceci a provoqué un débat où s'affrontent, sur le fond, ceux qui pensent que le RAC doit continuer à être un instrument permettant de contrôler les juges (Luis DíezPicazo) et ceux qui soutiennent qu'il devrait s'orienter davantage vers le contrôle du législateur (Francisco Rubio Llorente, Pedro Cruz Villalón). On pourrait dire que tous les problèmes relatifs à l'amparo (champ de protection, objet, recevabilité, principes directeurs) sont d'une façon ou d'une autre liés à cette question. La réforme de 2007 s'est penchée sur la seconde possibilité (amparo en tant que contrôle du législateur) pour justement essayer de minorer les tensions avec les juges ordinaires.
Le RAC est une institution qui a connu un succès retentissant ${ }^{146}$ : il a permis de donner un contenu aux droits fondamentaux; la doctrine ainsi créée a été suivie par les tribunaux (de bon gré, et pas uniquement à cause de l'obligation imposée à leur égard par l'article 5.1 de la loi organique $6 / 1985$ du pouvoir judiciaire); et, ce qui est encore plus important, il a contribué de manière décisive à créer une "culture des droits fondamentaux", non seulement dans une magistrature en bonne partie étrangère à ceux-ci, mais également dans la population.

À l'heure du bilan, si une chose est claire c'est que la surcharge actuelle en matière d'affaires est insoutenable. La réforme de 2007 n'était pas parvenue à diminuer le nombre d'affaires dans un premier temps (2007-2010), mais une réduction a pu être constatée en 2011. Depuis 2011, le nombre d'amparo reste stable autour de 7 200-7500 affaires. La réforme opérée en 2007 a été insuffisante, ce qui rend encore plus urgente et, à notre avis, inéluctable, l'introduction du modèle de certiorari pour juger de la recevabilité des affaires. Cependant, la perspective de cette réforme est devenue problématique depuis la décision de la Cour européenne des droits de l'homme dans l'affaire Arribas Antón c. Espagne.

La question de la réforme du champ de protection du $\mathrm{RAC}$ reste ouverte. À partir du moment où la réforme de 2007 a choisi un modèle d'amparo « contrôle du législateur» à la place du précédent amparo "contrôle des juges", la position de ceux qui, tout en défendant le nouveau modèle de 2007, prônent le maintien de l'article 24 de la Constitution (droit au procès équitable) dans le champ d'application de l'amparo constitutionnel (avec comme argument que l'article 24 de la Constitution garantit aussi un droit à ce que le juge applique dans le cas la loi en vigueur) n'est plus tenable ${ }^{147}$.

Après le choix d'un amparo « contrôle du législateur", il faudrait désormais se poser la question de savoir s'il ne serait pas nécessaire, pour mieux atteindre cet objectif, d'élargir l'objet du RAC pour rendre possibles des recours contre les lois.

Tout ce qui précède ne préjuge cependant en rien du choix final du modèle (contrôle du législateur ou du juge) qui répond fondamentalement à un modèle politique. Certes, à notre avis, il existe déjà des mécanismes pour contrôler le législateur (recours d'inconstitutionnalité des lois, renvoi judiciaire sur la constitutionnalité des lois). En

143. Voir F. Rubio Llorente, réponse à «Problemas actuales del recurso de amparo", Anuario de derecho constitucional y parlamentario, $\mathrm{n}^{\circ}$ 2, 199o, p. 158-159; R. L. Blanco Valdés, «La política y el derecho... », p. 262-263.

144. B. de la Cuadra, «El Tribunal Supremo apela al poder moderador del Rey contra un fallo del Constitucional», El País, 4 février 1994, en ligne: https://elpais.com/diario/1994/o2/o4/espana/760316428_850215.html.

145. Le texte intégral de la décision est le suivant: "Dans les procès au pénal pour lesquels a été rendu un arrêt de cassation annulé par le Tribunal constitutionnel dans le cadre d'un recours d'amparo pour atteinte aux droits fondamentaux, la deuxième chambre du Tribunal suprême, organe juridictionnel supérieur de l'ordre pénal, devra nécessairement conclure le procès en rendant l'arrêt correspondant dans les termes qui auraient été pertinents s'il avait été fait droit en cassation à l’atteinte appréciée par le Tribunal constitutionnel, donc avec la nullité et les effets décidés par le Tribunal constitutionnel».

146. Depuis les 280 recours introduits la première année de fonctionnement du tribunal (1980) jusqu'à un maximum de 10792 en 2009 . En 2015 , 7203 amparo.

147. Voir M. Aragón Reyes, «La reforma... », p. 36-38. 
revanche, la configuration du RAC «contrôle des juges » répond, selon nous, à une logique politique implacable. La Constitution proclame, en effet, que «[1]a souveraineté nationale réside dans le peuple espagnol duquel émanent tous les pouvoirs de l'État $»^{148}$ et que " [l]a justice émane du peuple et est administrée [...] par des juges et des magistrats $[. .$.$] responsables { }^{149}$. Dans un État démocratique, il ne peut y avoir de pouvoirs politiquement irresponsables. En Espagne, néanmoins, les juges se trouvent soumis à une responsabilité civile, pénale et disciplinaire, mais l'autocontrôle n'est pas un véritable contrôle. Ils ne sont pas soumis à une responsabilité politique (comme dans les systèmes anglo-saxons). L'amparo "contrôle des juges" est l'unique forme de contrôle politique d'un pouvoir qui échappe à la logique démocratique : le Tribunal constitutionnel est, en fin de compte, un organe politique même s'il possède un pouvoir juridictionnel. Cependant, l'abandon au niveau législatif de cette option entraîne un certain déséquilibre du système des contrôles pour l'ensemble de l'État. 